\title{
Capacity Improvement in CDMA Downlink With Orthogonal Code-Hopping Multiplexing
}

\author{
Sung Ho Moon, Student Member, IEEE, Suwon Park, Member, IEEE, Jae Kyun Kwon, Member, IEEE, and
} Dan Keun Sung, Senior Member, IEEE

\begin{abstract}
Orthogonal code-hopping multiplexing (OCHM) is a statistical multiplexing scheme designed to increase the number of allowable downlink channels in code division multiple access (CDMA) systems. OCHM is expected to compensate for a lack of codewords in future communication systems. In CDMA systems including OCHM, system capacity is limited by the number of codewords and power (or interference), and the maximum system capacity is determined by a stronger limitation between them. Call blockings due to power limitation may occur firstly if downlink channels demand large $E_{\mathrm{b}} / I_{0}$ values and a high-channel activity. On the other hand, code limitation may occur prior to power limitation in CDMA. The maximum system capacities determined by both code and power limitations must be known, even in OCHM. However, previous studies on OCHM system capacity focused only on increasing the number of multiplexed users with no consideration of the power limitation. In this paper, the overall system capacity of OCHM considering both code and power limitations was evaluated. For this analysis, the transmission chip energy of base station (BS) and inner/outer-cell interference is mathematically derived in a multicell and multiuser environment. The downlink system capacity for OCHM is larger than for orthogonal code division multiplexing (OCDM) as other cell interference (OCI), mean channel activity, and the required $E_{\mathrm{b}} / I_{0}$ value decrease.
\end{abstract}

Index Terms-Code division multiple access (CDMA), downlink capacity, orthogonal code-hopping multiplexing (OCHM), perforation, synergy.

\section{INTRODUCTION}

$\mathbf{P}$ ACKET-TYPE services, such as hypertext transfer protocol (HTTP), file transfer protocol (FTP), and wireless application protocol (WAP), have gradually increased and may become dominant in future wireless communications. In contrast to voice traffic, which is a major service in $2 \mathrm{G}$, these packet-based services have two different characteristics. First, they exhibit high burstiness with low activity and second,

Manuscript received October 25, 2004; revised April 24, 2005 and July 12, 2005. This work was supported in part by MIC, Korea, under the BrOMA Information Technology Research Center (ITRC) support program supervised by Institute of Information Technology Assessment (IITA). The review of this paper was coordinated by Prof. T. Lok.

S. H. Moon and D. K. Sung are with the Department of Electrical Engineering and Computer Science, Korea Advanced Institute of Science and Technology (KAIST), Daejeon 305-701, Korea (e-mail: dksung@ee.kaist.ac.kr).

S. Park was with the Department of Electrical Engineering and Computer Science, Korea Advanced Institute of Science and Technology (KAIST), Daejeon 305-701, Korea. He is now with the Department of Electronics and Communications Engineering, Kwangwoon University, Seoul 139-701, Korea (e-mail: spark@ieee.org).

J. K. Kwon was with the Department of Electrical Engineering and Computer Science, Korea Advanced Institute of Science and Technology (KAIST), Daejeon 305-701, Korea. He is now with Electronics and Telecommunications Research Institute (ETRI), Daejeon 305-701, Korea.

Digital Object Identifier 10.1109/TVT.2005.863468 packet-based traffic is generally concentrated on the downlink since mobile users often demand information from servers. Based on these characteristics, demands for downlink code channels will increase more for packet-based services in $3 \mathrm{G}$ and $4 \mathrm{G}$ systems than for voice traffic. However, orthogonal code allocation/deallocation mechanisms in conventional code division multiple access (CDMA) systems are inefficient for serving the bursty downlink packet-type traffic since code channels are allocated to mobile stations (MSs) by a base station (BS) at each initial call setup, and are released from the connection after each call termination. Waste of orthogonal code channels due to many inactive periods of bursty traffic and increased demands for orthogonal code channels may result in a lack of orthogonal codewords (OCs) in spite of remaining BS power.

Orthogonal code-hopping multiplexing (OCHM) [1] can solve the problem of efficient multiplexing of data packets. Systems based on OCHM can accommodate more downlink channels than the number of OCs by using statistical multiplexing. This means that OCHM can compensate for a lack of OCs without significant changes in conventional systems. In CDMA $3 \mathrm{G}$ and $4 \mathrm{G}$ packet services, more OCs may be needed. A multiscrambling code for wideband code division multiple access (W-CDMA) [2] and a quasi-orthogonal function for cdma2000 [3] were proposed to alleviate this code shortage. However, these techniques result in a loss of perfect orthogonality between codewords. Orthogonality is a valuable property of synchronous downlink since it yields a natural cancellation of interference. Thus, OCHM is an attractive multiplexing scheme.

There are two limitations in CDMA downlink for determination of the maximum system capacity. First, the number of connecting MSs in a code-limited system cannot exceed the number of OCs available in the system. Second, in a power (or interference) limited system like 2G CDMA, the number of voice users is limited by interference due to mean channelactivity values of $0.4 \sim 0.5$. Thus, the maximum allowable number of users is less than the number of OCs. There have been previous studies regarding the maximum system capacity in power-limited CDMA downlinks. The maximum number of simultaneous users with a downlink pole equation was analyzed in [4] and [5] and the downlink Erlang capacity and outage probability were evaluated in [6].

In previous studies [7]-[11] on OCHM, capacity analysis was only focused on code capacity, which is defined as the maximum allowable number of users limited by the number of codewords under the assumption that system has no power limitation. Since a power shortage rarely occurs prior to a lack 
of codewords for packet services with relatively low-channel activities, the code capacity and bit-error-rate (BER) performance were evaluated and compared with conventional multiplexing schemes. However, system capacity may be also limited by power (or interference) even if downlink channels experience low-mean channel activities. Therefore, power capacity, which is defined as the maximum allowable number of users limited by the maximum transmission power of a BS under the assumption that system has no code limitation, should also be evaluated in OCHM because the overall system capacity is a minimum value between code and power capacities.

In this paper, we briefly introduce OCHM based on previous studies and evaluate the capacity improvement in OCHM-based downlink considering both code and power capacities. The effect of an increase in code capacity and OCHM link performance has already been evaluated by mathematical analysis and simulation [1]. Based on these previous results, the power capacity of OCHM is mathematically analyzed based on the maximum number of users, and the overall system capacity is obtained, and numerical examples for the code and power capacities are shown.

The rest of this paper is organized as follows: the previously proposed OCHM scheme is briefly introduced in Section II. Notations for analysis are summarized in Section III. In Section IV, we review a system capacity improvement in code capacity in OCHM. The power capacity of OCHM is evaluated in Section V, and numerical examples of the analysis are shown in Section VI. Finally, conclusions and further studies are presented in Section VII.

\section{OCHM}

Several previous studies have analyzed code-hopping schemes in spread spectrum systems [12]-[14]. These previous studies focused mainly on showing that code-hopping systems exhibit better BER performance than conventional spread spectrum systems because cochannel interference is averaged with time diversity. However, OCHM [1] is distinguished from these previous code-hopping methods because code-hopping in OCHM is used for downlink multiplexing in order to increase the utilization of code channels. Moreover, OCHM is only applicable to the downlink because a BS knows all downlink data transmitted to all MSs in its cell.

Fig. 1 shows the basic operations of OCHM and the conventional downlink multiplexing scheme, here called orthogonal code division multiplexing (OCDM). In the conventional OCDM [Fig. 1(a)], each modulation symbol during a modulation symbol time $T_{\mathrm{S}}$ is spread by a specific OC. During a call for each MS, an OC allocated to the MS is maintained. The MSs indexed from \#a to \#g maintain only one of the OCs indexed from \#A to \#G, regardless of the inactive periods for MS\#c and MS\#g. Thus, many OC resources are wasted in a bursty traffic environment.

On the other hand, OCHM allows change of the OCs in every modulation symbol time $T_{\mathrm{s}}$ and multiplexing of a larger number of MSs than the number of OCs. For example, in Fig. 1(b), MS \#f changes an OC for each modulation symbol based on its hopping pattern (HP) indexed by \#f. An MS-specific HP can be generated based on the MS identifier (ID), such as an electronic serial number (ESN) at an initial channel allocation time.

From the random hopping of OCs, the BS can multiplex more downlink channels than the number of OCs. However, code collisions between codewords are inevitable with higher utilization of OCs. Fortunately, the BS can monitor all information on the downlink channels. The BS compares each channel to all other channels during multiplexing to determine which symbols collide and deals with the colliding symbols before transmission.

When a code collision occurs among the HPs of active downlink channels, a comparator and controller in the OCHM transmitter performs one of the following two operations: perforation or synergy.

1) Perforation: If code-collision symbols do not have an identical symbolic value, i.e., +1 or 0 for binary phaseshift keying (BPSK), then none of the data symbols colliding during a symbol time of $T_{\mathrm{S}}$ are transmitted.

2) Synergy: If code-collision symbols have an identical symbolic value, the data symbols colliding during a symbol time of $T_{\mathrm{s}}$ are transmitted and the transmission signal amplitude of the OC during the symbol time is the sum of the signal amplitudes assigned to all of the corresponding downlink channels.

This perforation/synergy scheme for code collision in a codehopping system is a unique concept. Fig. 2 shows the resultant, transmitted signal after perforation and synergy for MS\#f. One of the code collisions related to OC\#A from $(n+2) T_{\mathrm{s}}$ to $(n+3) T_{\mathrm{s}}$ is managed by a perforation, and there is no transmission. For the other interval from $(n+7) T_{\mathrm{s}}$ to $(n+8) T_{\mathrm{s}}$, the code collision experiences a synergy, and an additive signal of the two symbols due to the synergy effect is transmitted.

Regardless of perforated symbols, the channel decoder in the receiver of the corresponding MS can recover the transmitted data. The synergy operation allows the codecollision symbols to maintain and share their transmission powers in common. Thus, the synergy operation results in a transmission power gain.

\section{Notations For MATHEMAtical AnAlysis}

$(i, b) \quad i$ th MS in the $b$ th cell.

$(i, j, b) \quad i$ th MS scrambled by the $j$ th scrambling code in the $b$ th cell.

$\alpha_{(i, 0, b)} \quad$ Correlation factor of OCs at MS $(i, 0, b)$.

$\bar{\alpha} \quad$ Mean correlation factor of OCs.

$b \quad$ Index of a cell or a BS.

$d \quad$ Distance between a BS and an adjacent BS.

$\epsilon \quad$ MS closer than $\epsilon$ are considered to be $\epsilon$-distant from the BS [15].

$E_{\mathrm{c}, b}^{(\mathrm{t})} \quad$ Total transmission chip energy of BS $b$.

$E_{\mathrm{c}, \max }^{(\mathrm{t})} \quad$ Maximum downlink transmission chip energy.

$E_{\mathrm{c}, b \rightarrow m}^{(\mathrm{t})} \quad$ Transmission chip energy from BS $b$ to MS $\underline{m}$.

$E_{\mathrm{c}, b \rightarrow \underline{m}}^{(\mathrm{r})} \quad$ Received chip energy from BS $b$ to MS $\underline{m}$.

$\gamma \quad$ Propagation loss factor $(=4)$.

$I_{0, i, 0, b)} \quad$ Total interference chip energy at MS $(i, 0, b)$.

$I_{\mathrm{ic},(i, 0, b)} \quad$ Inner-cell interference chip energy at MS $(i, 0, b)$. 


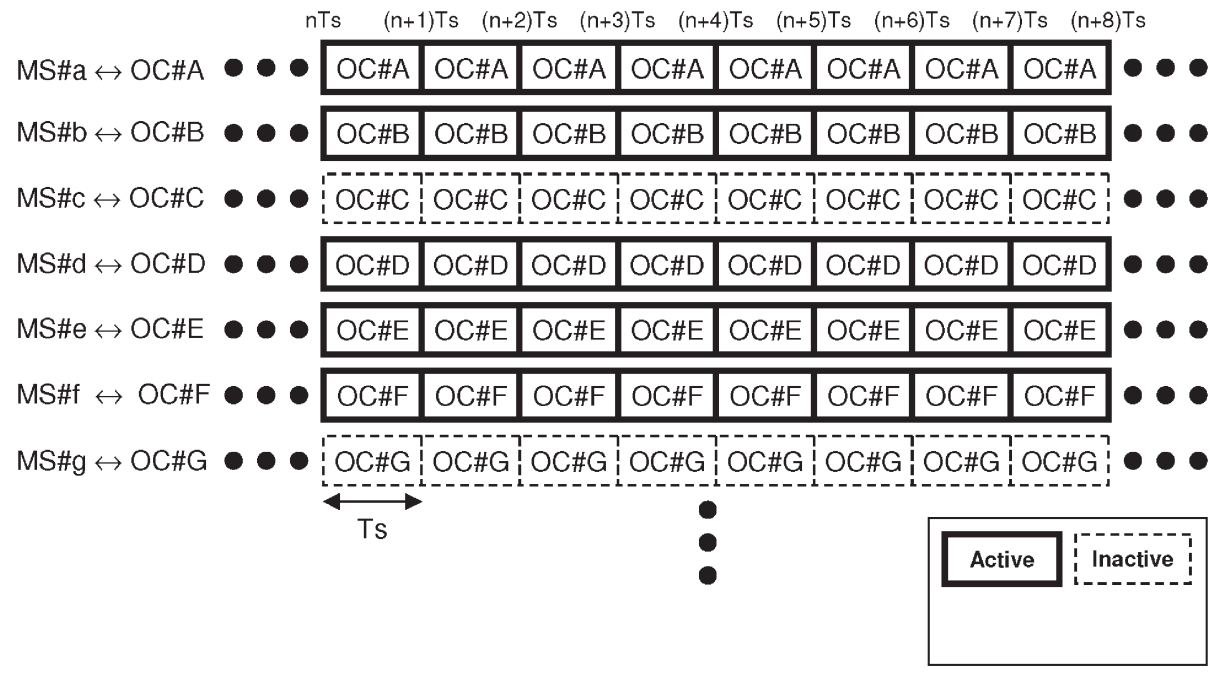

(a)

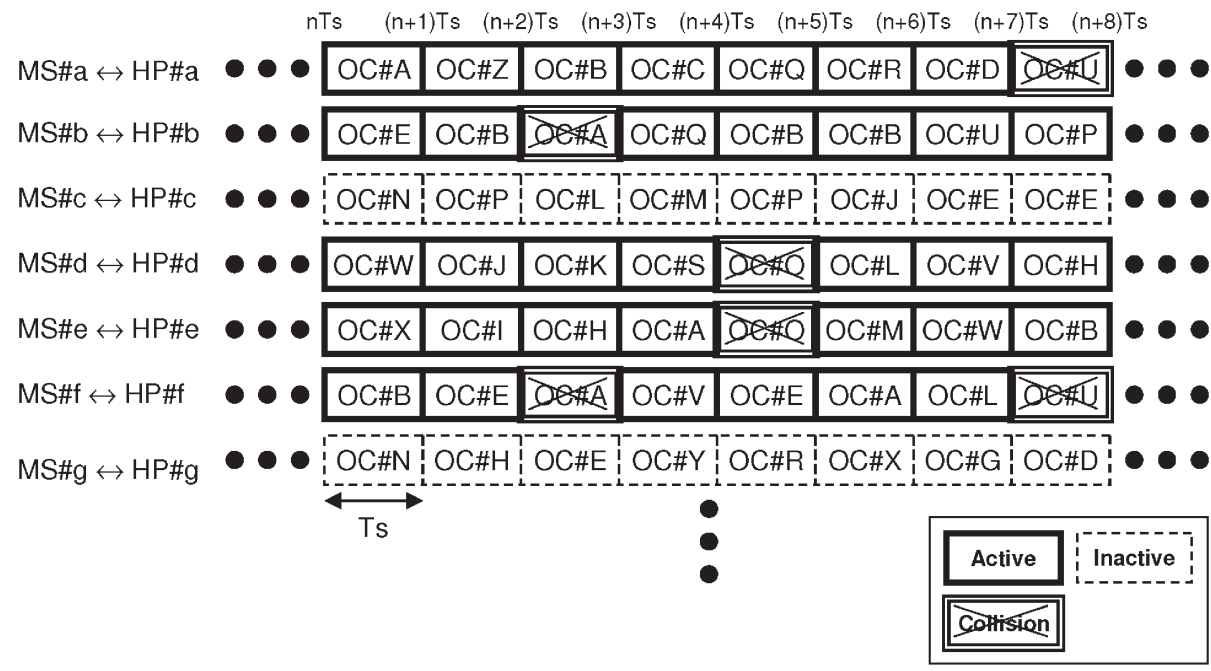

(b)

Fig. 1. Basic operations of (a) OCDM and (b) OCHM.

$I_{\mathrm{oc},(i, 0, b)} \quad$ Outer-cell interference chip energy at MS $(i, 0, b)$.

$\underline{m} \quad$ Index of an MS, where $\underline{m}=(i, b)$ or $(i, j, b)$.

$M_{b}^{\mathrm{c}} \quad$ Code capacity in the $b$ th cell.

$M_{b}^{\mathrm{p}} \quad$ Power capacity in the $b$ th cell.

$N_{0} \quad$ One-sided power spectral density of additive white Gaussian noise (AWGN).

$N_{\text {OC }} \quad$ Number of OCs for the downlink.

$\nu_{b \rightarrow \underline{m}} \quad$ Channel activity of a downlink channel from BS $b$ to MS $m$.

$\bar{\nu} \quad$ Mean channel activity of downlink channels.

$p_{\mathrm{c}, \mathrm{OC}} \quad$ Code-collision probability.

$p_{\mathrm{p}, \mathrm{OC}} \quad$ Perforation probability.

$p_{\mathrm{s}, \mathrm{OC}} \quad$ Synergy probability.

$r_{b \rightarrow \underline{m}} \quad$ Distance from BS $b$ to $\mathrm{MS} \underline{m}$.

$\rho \quad$ Proportion of maximum downlink transmit power for downlink common control channels.

$r_{\mathrm{e}} \quad$ Equivalent cell radius of a hexagonal cell.

$r_{\mathrm{h}} \quad$ Radius of a circumcircle surrounding a hexagonal cell.

$R_{1} \quad$ Random variable representing the distance between a BS and an MS in a cell covered by the BS.
$R_{2} \quad$ Random variable representing the distance from a $\mathrm{BS}$ and an MS in an adjacent cell of the cell covered by the BS.

$R_{m}^{\mathrm{FEC}} \quad$ Channel (or forward error correction) coding rate of downlink channel for MS $\underline{m}$.

$s \quad$ Number of possible constellation points (i.e., $s=2$ for BPSK).

Transmission antenna main lobe width $\left(\theta=120^{\circ}\right.$ for three-sectorized cell).

Shadow fading from BS $b$ to MS $\underline{m}$.

\section{CODE CAPACITY OF OCHM}

As defined in Section I, code capacity is obtained under an assumption that there is no power limitation. In OCDM, if there are $N_{\text {OC }}$ codewords, $N_{\text {OC }}$ MSs can maintain maximal connections. The $\left(N_{\mathrm{OC}}+1\right)$ th connection attempt is not permitted even if most connections are inactive. Therefore, code capacity in OCDM is always fixed to $N_{\text {OC }}$. However, the code capacity in OCHM can increase more than $N_{\text {OC }}$ depending on the perforation (or code collision) probability. 


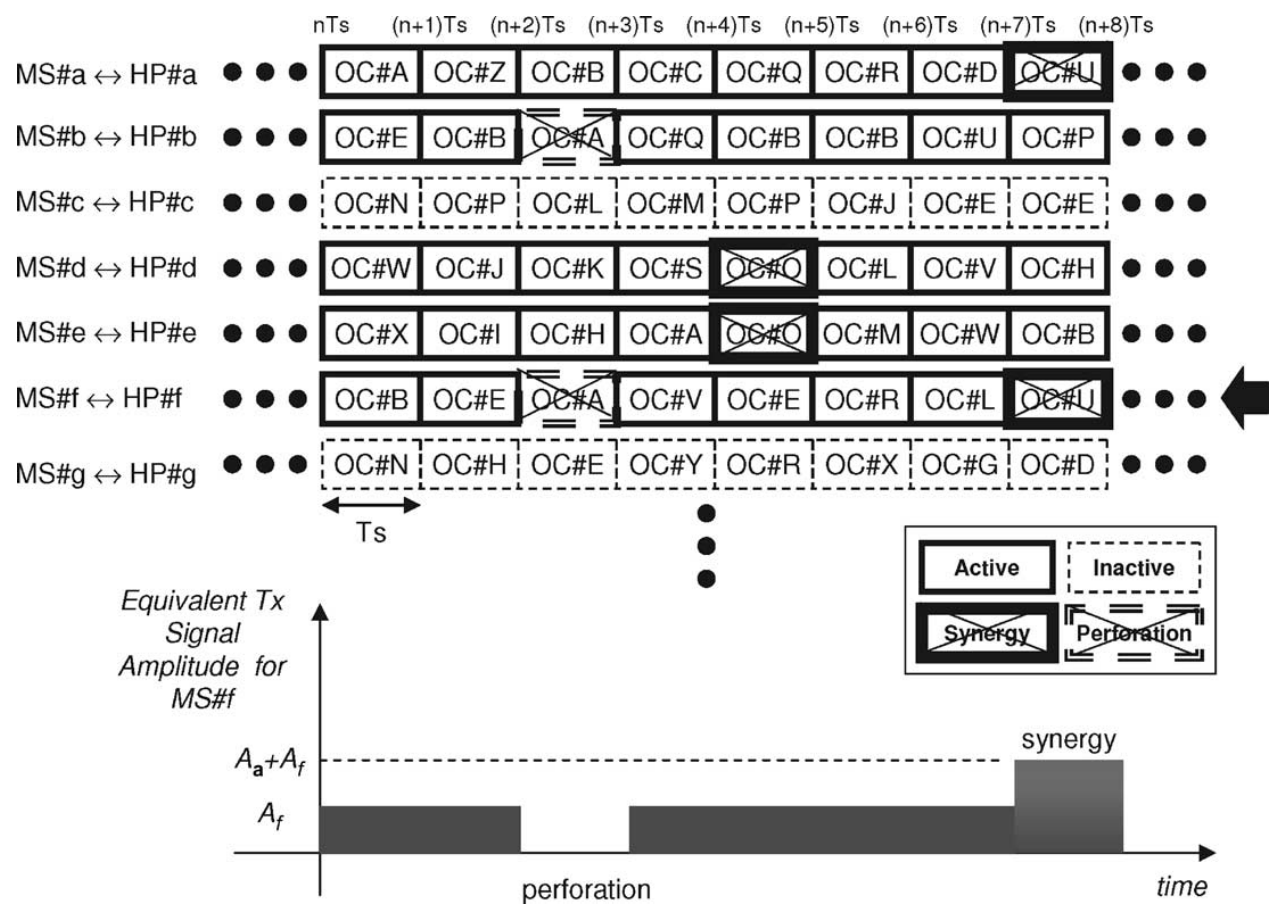

Fig. 2. Downlink transmission signal for MS\#f.

For a given number of allocated orthogonal downlink channels and available number of codewords, the more active the allocated channels are, the more often the downlink channels may experience code collisions. Therefore, for each modulation symbol, we have the following probabilities [8]:

$$
\begin{aligned}
& p_{\mathrm{c}, \mathrm{OC}}=1-\left(1-\frac{\bar{\nu}}{N_{\mathrm{OC}}}\right)^{M_{b}^{\mathrm{c}}-1} \\
& p_{\mathrm{p}, \mathrm{OC}}=1-\left(1-\frac{s-1}{s} \cdot \frac{\bar{\nu}}{N_{\mathrm{OC}}}\right)^{M_{b}^{\mathrm{c}-1}} \\
& p_{\mathrm{s}, \mathrm{OC}}=p_{\mathrm{c}, \mathrm{OC}}-p_{\mathrm{p}, \mathrm{OC} .}
\end{aligned}
$$

Given $p_{\mathrm{p}, \mathrm{OC}}$, the code capacity in OCHM is obtained as

$$
M_{b}^{\mathrm{c}}=1+\frac{\ln \left(1-p_{\mathrm{p}, \mathrm{OC}}\right)}{\ln \left(1-\frac{(s-1) \cdot \bar{\nu}}{s \cdot N_{\mathrm{OC}}}\right)} .
$$

As shown in (4), the code capacity is determined by the perforation probability. With a larger perforation probability, more downlink channels can be accommodated for a given mean channel-activity factor. Therefore, "code limited" in OCHM can be also called "perforation limited."

Fig. 3 shows the code capacity for a given allowable perforation probability $p_{\mathrm{p}, \mathrm{OC}}$ with $64 \mathrm{OCs}$ and QPSK modulation. In case of QPSK, we can set $s=2$ from the independence between $I$ - and $Q$-axes. If we have no limitation in the transmission power, OCHM increases code capacity up to 287,370 , and 458 with a mean channel activity of 0.1 and $p_{\mathrm{p}, \mathrm{OC}}$ values of $0.2,0.25$, and 0.3 , respectively.

The allowable perforation probability for code capacity cannot increase infinitely because a larger allowable perforation probability requires a larger $E_{\mathrm{b}} / I_{0}$ value. Therefore, from linklevel simulation, we can determine a proper allowable perfora- tion probability to satisfy a link quality. Fig. 4 [1] shows the frame-error-rate (FER) performance of OCHM in an AWGN channel. The FER performance of OCHM with convolutional coding (CC) and turbo coding (TC) is evaluated by simulation. The code rate is $1 / 4$, and all parameters follow the cdma2000 specification [3]. The curve of $p_{\mathrm{p}, \mathrm{OC}}=0.00$ corresponds to the conventional OCDM scheme without perforation. The result shows that additional transmission power is a maximum of $1 \mathrm{~dB}$ compared to OCDM in order to meet a target FER value of 0.01. From Figs. 3 and 4, OCHM enhances the code capacity from 64 to 370 with a slightly increased $E_{\mathrm{b}} / I_{0}$ value of $0.5 \mathrm{~dB}$ for a mean channel-activity factor of 0.1 and an FER value of 0.01 with TC.

\section{POWER CAPacity OF OCHM}

In this section, we analyze the power capacity of OCHM downlink channels in a cell, and assume that there is no code (or perforation) limitation. In addition, soft and softer handovers are not considered because we analyze the power capacity of packet services with relatively high burstiness. It is generally known that hard handoffs are more efficient for packet traffic than are soft and softer handoffs.

\section{A. Constraint for Estimating Power Capacity}

Fig. 5 shows a CDMA system consisting of one inner and six outer cells. CDMA systems are generally known to be limited by power (or interference). Since the maximum downlink transmission power is limited to avoid interference with MSs in adjacent cells, downlink channels need to be admitted within the maximum downlink transmission power. Therefore, a main constraint in downlink is that a BS can transmit within the maximum downlink transmission power. Since the chip 


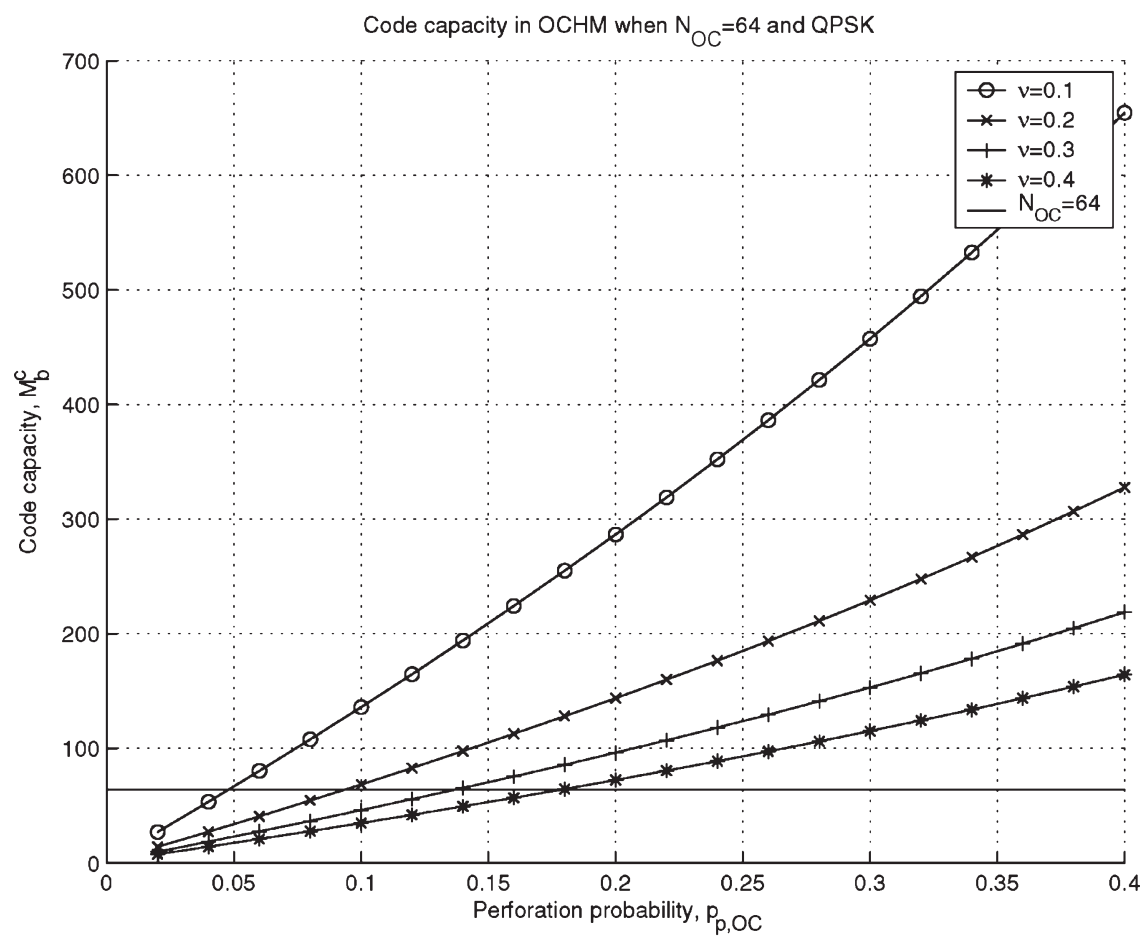

Fig. 3. Code capacity in OCHM.

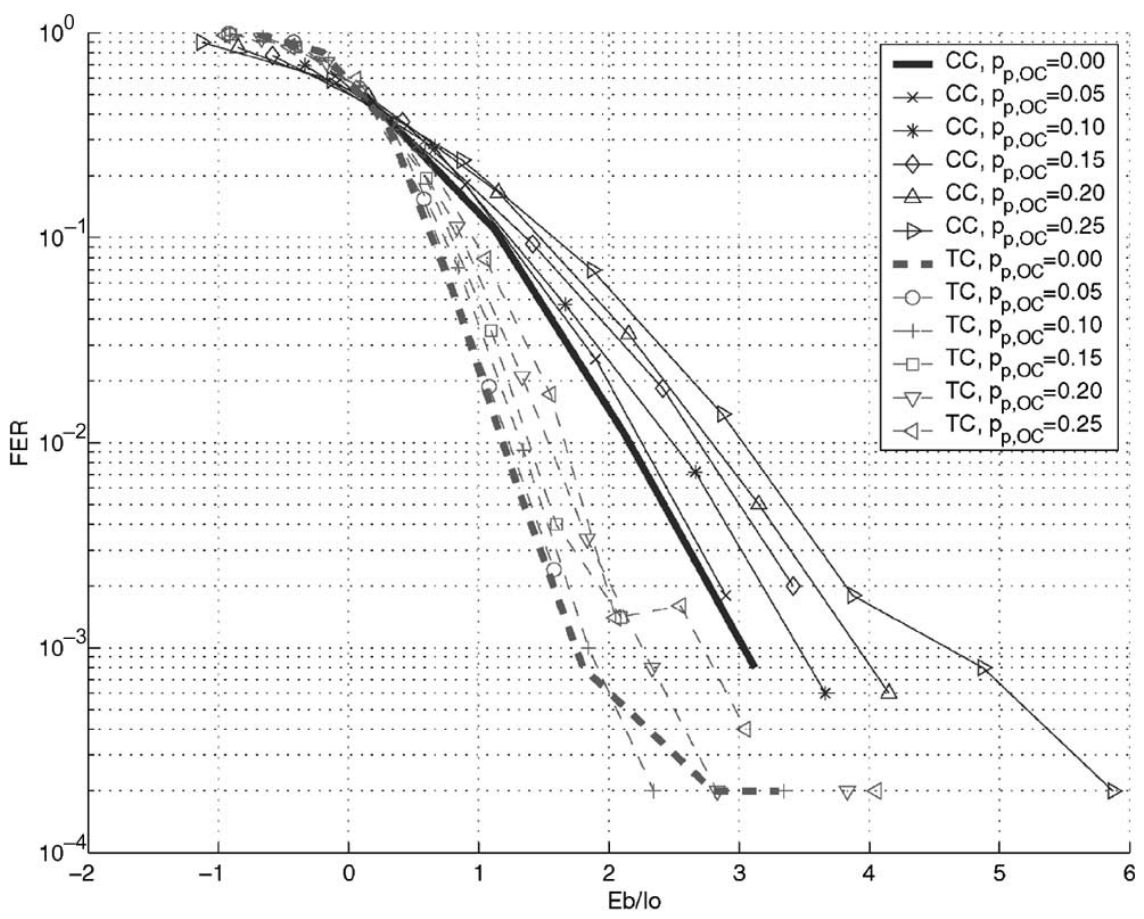

Fig. 4. FER versus $E_{\mathrm{b}} / I_{0}$ in AWGN.

duration $T_{\mathrm{c}}$ is fixed, the chip energy is proportional to power. Thus, the total transmission chip energy of BS $b$ must satisfy the following condition:

$$
E_{\mathrm{c}, b}^{(\mathrm{t})}=\rho E_{\mathrm{c}, \max }^{(\mathrm{t})}+\sum_{\underline{m}} \nu_{b \rightarrow \underline{m}} E_{\mathrm{c}, b \rightarrow \underline{m}}^{(\mathrm{t})} \leq E_{\mathrm{c}, \max }^{(\mathrm{t})}
$$

where $\rho$ is the energy portion for downlink broadcasting channels (typically, $\rho=0.1 \sim 0.3$ ) [16].

Equation (5) is a requirement to determine the power capacity in CDMA systems. Thus, our final goal here is to find the number of downlink connections accommodated in a given condition using this inequality. 


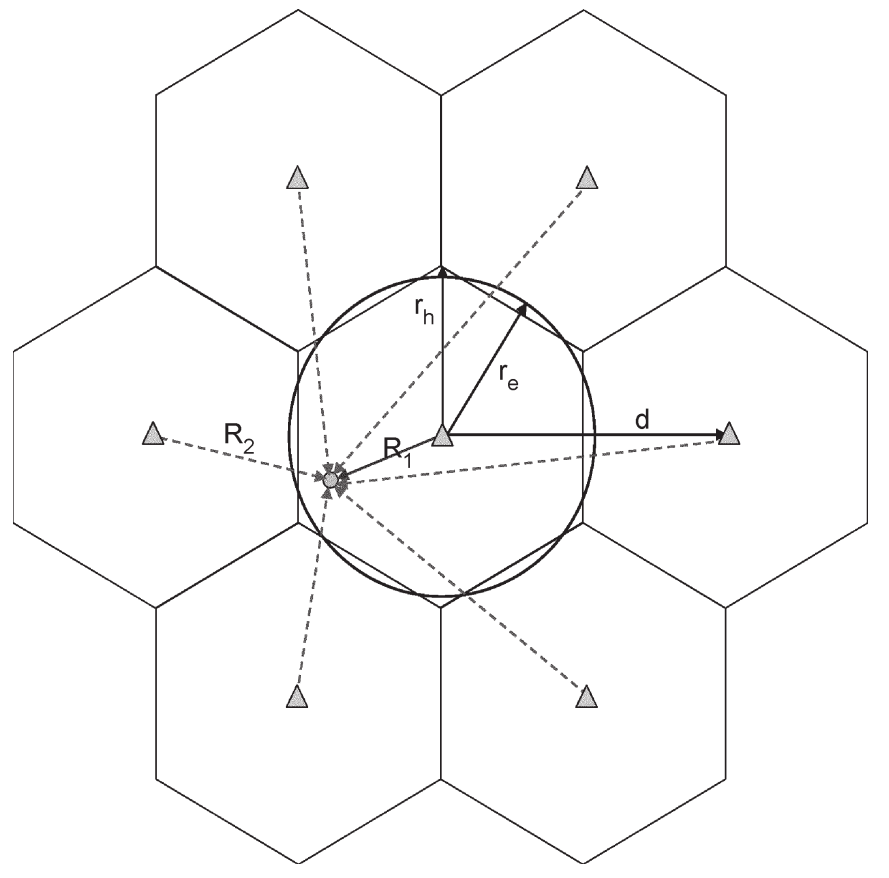

Fig. 5. One inner cell and six outer cells.

\section{B. Wireless Channel: Propagation Loss and Shadowing}

For simplicity of analysis, we consider only propagation loss and shadow fading. Short-term fading due to the movement of an MS is not considered. Short-term fading is assumed to be averaged out by fast power control. In real environments, a BS requires additional transmission power in order to overcome short-term fading.

In order to express (5) in terms of the number of downlink connections, we must know the average transmission chip energy and the channel activity per MS. Under an assumption of perfect power control, the average transmission chip energy per MS is determined depending on the required $\left(E_{\mathrm{c}} / I_{0}\right)_{\text {target }}$ value in receiver. The received chip energy at MS $\underline{m}$ is given as

$$
E_{\mathrm{c}, b \rightarrow \underline{m}}^{(\mathrm{r})}=E_{\mathrm{c}, b \rightarrow \underline{m}}^{(\mathrm{t})} \cdot r_{b \rightarrow \underline{m}}^{-\gamma} \cdot 10^{\frac{-\zeta_{b \rightarrow \underline{m}}}{10}} .
$$

The propagation loss is closely related to the distance between a transmitter and a receiver. We consider two distances, as shown in Fig. 5. Distance $R_{1}$ is between a BS and an MS in a cell covered by the BS, and distance $R_{2}$ is between a BS and an MS in a cell adjacent to the cell covered by the BS.

When MSs are uniformly distributed in a cell, the probability density functions for $R_{1}$ and $R_{2}$ are derived as

$$
\begin{aligned}
& f_{R_{1}}(r)=\frac{2 r}{r_{\mathrm{e}}^{2}} \\
& f_{R_{2}}(r) \simeq \frac{2}{\sqrt{3}} \cdot \frac{\sqrt{4 d^{2} r_{\mathrm{e}}^{2}-\left(d^{2}+r_{\mathrm{e}}^{2}-r^{2}\right)^{2}}}{d^{3}} .
\end{aligned}
$$

The detailed derivation of (8) is given in the Appendix.
If MS $\underline{m}$ is served by a BS of index $b$, from (7), the $\gamma$ th moment of distance $R_{1}$ from $\mathrm{BS} b$ to $\mathrm{MS} \underline{m}$ is derived as

$$
E\left[r_{b \rightarrow \underline{m}}^{\gamma}\right]=\int_{\varepsilon}^{r_{\mathrm{e}}} \frac{2 r^{\gamma+1}}{r_{\mathrm{e}}^{2}} d r \simeq \frac{2}{\gamma+2}\left(r_{\mathrm{e}}^{\gamma}-\varepsilon^{\gamma}\right)
$$

where $\epsilon \simeq 10[\mathrm{~m}]$.

When MS $\underline{m}$ is not served by BS $b$, the $\gamma$ th moment of $r_{z \rightarrow \underline{m}}(z \neq b)$ using $(8)$ is derived as

$$
E\left[r_{z \rightarrow \underline{m}}^{\gamma}\right]=\int_{(\sqrt{3}-1) r_{\mathrm{e}}}^{(\sqrt{3}+1) r_{\mathrm{e}}} \frac{2 r^{\gamma}}{\sqrt{3}} \cdot \frac{\sqrt{4 d^{2} r_{\mathrm{e}}^{2}-\left(d^{2}+r_{\mathrm{e}}^{2}-r^{2}\right)^{2}}}{d^{3}} d r
$$

where $r_{\mathrm{e}} \simeq r_{\mathrm{h}}=d / \sqrt{3}$.

Typically, shadowing in a wireless channel is modeled as a log-normal distribution with zero mean. Thus, the expectation of a log-normally distributed random variable with a mean of $m_{\zeta}=0$ and a variance of $\sigma_{\zeta}^{2}$ is written as

$$
\begin{aligned}
E\left[10^{\left.\frac{-\zeta_{b \rightarrow \underline{m}}}{10}\right]}\right. & =10^{\frac{-m_{\zeta}}{10}} \cdot \exp \left\{\frac{\left(\frac{\ln 10}{10} \sigma_{\zeta}\right)^{2}}{2}\right\} \\
& =\exp \left\{\frac{\left(\frac{\ln 10}{10} \sigma_{\zeta}\right)^{2}}{2}\right\} .
\end{aligned}
$$

\section{Total Transmission Chip Energy From BS b}

If we assume that the required $\left(E_{\mathrm{c}} / I_{0}\right)_{\text {target }}$ value in receiver is determined for an MS, the transmission chip energy per MS from a BS can be obtained. We assume that MSs have three dimensional indexes such as $\underline{m}=(i, 0, b)$. MS $(i, j, b)$ is the $i$ th MS scrambled by the $j$ th scrambling code in a cell covered by a BS of index $b$. For simplicity of mathematical analysis, we assume that only one scrambling code is used $(j=0)$. Then

$$
E_{\mathrm{c}, b \rightarrow(i, 0, b)}^{(\mathrm{t})}=E_{\mathrm{c}, b \rightarrow(i, 0, b)}^{(\mathrm{r})} \cdot r_{b \rightarrow(i, 0, b)}^{\gamma} \cdot 10^{\frac{\zeta_{b \rightarrow(i, 0, b)}}{10}} .
$$

The required chip energy at the MS $(i, 0, b)$ is calculated as a multiplication of $\left(E_{\mathrm{c}} / I_{0}\right)_{\text {target }}$ and $I_{0,(i, 0, b)}$. Thus, the total transmission chip energy from BS $b$ is expressed as

$$
\begin{aligned}
E_{\mathrm{c}, b}^{(\mathrm{t})}=\rho E_{\mathrm{c}, \max }^{(\mathrm{t})}+\sum_{(i, 0, b)}\left(\nu_{b \rightarrow(i, 0, b)} \cdot E_{\mathrm{c}, b \rightarrow(i, 0, b)}^{(\mathrm{t})}\right) \\
=\rho E_{\mathrm{c}, \max }^{(\mathrm{t})}+\sum_{(i, 0, b)}\left(\nu_{b \rightarrow(i, 0, b)} \cdot E_{\mathrm{c}, b \rightarrow(i, 0, b)}^{(\mathrm{r})}\right. \\
\left.\cdot r_{b \rightarrow(i, 0, b)}^{\gamma} \cdot 10^{\left.\frac{\zeta_{b \rightarrow(i, 0, b)}}{10}\right)}\right) \\
=\rho E_{\mathrm{c}, \max }^{(\mathrm{t})}+\sum_{(i, 0, b)}\left(\nu_{b \rightarrow(i, 0, b)} \cdot\left(\frac{E_{\mathrm{c}}}{I_{0}}\right)_{\mathrm{target}} \cdot I_{0,(i, 0, b)}\right. \\
\left.\cdot r_{b \rightarrow(i, 0, b)}^{\gamma} \cdot 10^{\frac{\zeta_{b \rightarrow(i, 0, b)}}{10}}\right)
\end{aligned}
$$




\section{Interference at $M S(i, 0, b)$}

For complete derivation of (13), $I_{0,(i, 0, b)}$ must be calculated. The total interference chip energy at an MS is the sum of the inner-cell interference chip energy, the outer-cell interference chip energy, and the thermal noise. In (9) and (10), we calculate the expectations of propagation losses from a home cell and an adjacent cell. Thus, all received power from BSs can be expressed, and inner/outer-cell interference chip energy can be calculated. Since a BS has a maximum transmission chip energy of $E_{\mathrm{c}, \max }^{(\mathrm{t})}$, the total interference chip energy at MS $(i, 0, b)$ is bounded as

$$
\begin{aligned}
I_{0,(i, 0, b)}= & I_{\mathrm{ic},(i, 0, b)}+I_{\mathrm{oc},(i, 0, b)}+N_{0} \\
\leq & E_{\mathrm{c}, \max }^{(\mathrm{t})} \cdot r_{b \rightarrow(i, 0, b)}^{-\gamma} \cdot 10^{\frac{-\zeta_{b \rightarrow(i, 0, b)}}{10}} \\
& +\sum_{z \neq b} E_{\mathrm{c}, \max }^{(\mathrm{t})} \cdot r_{z \rightarrow(i, 0, b)}^{-\gamma} \cdot 10^{\frac{-\zeta_{z \rightarrow(i, 0, b)}}{10}}+N_{0} \\
= & E_{\mathrm{c}, \max }^{(\mathrm{t})}\left(r_{b \rightarrow(i, 0, b)}^{-\gamma} \cdot 10^{\frac{-\zeta_{b \rightarrow(i, 0, b)}}{10}}\right. \\
& \left.\quad+\sum_{z \neq b} r_{z \rightarrow(i, 0, b)}^{-\gamma} \cdot 10^{\frac{-\zeta_{z \rightarrow(i, 0, b)}}{10}}\right)+N_{0}
\end{aligned}
$$

where the subscripts ic and oc denote the inner-cell and the outer-cell interference, respectively.

Inner-cell interference occurs from cross correlation among spreading codes in the downlink. Usually in the downlink of CDMA, if a perfectly orthogonal code set is used, i.e., Walsh codes, no inner-cell interference occurs. However, the multipath effect corrupts the perfect orthogonality. For modeling of this effect, we set $\alpha$ as a correlation factor. If the downlink from BS $b$ to MS $(i, 0, b)$ is perfectly orthogonal, i.e., $\alpha_{(i, 0, b)}=1$, then the inner-cell interference is 0 , or $I_{\mathrm{ic},(i, 0, b)}=0$.

Since the inner-cell interference chip energy cannot exceed the total transmission chip energy of BS $b$ denoted as $E_{\mathrm{c}, b}^{(\mathrm{t})}$, the inner-cell interference chip energy at MS $(i, 0, b)$ is expressed and bounded as

$$
\begin{aligned}
I_{\mathrm{ic},(i, 0, b)}= & \left(\rho E_{\mathrm{c}, \max }^{(\mathrm{t})}+\sum_{\{(x, 0, b) \mid x \neq i\}} \nu_{b \rightarrow(x, 0, b)} E_{\mathrm{c}, b \rightarrow(x, 0, b)}^{(\mathrm{t})}\right) \\
& \cdot r_{b \rightarrow(i, 0, b)}^{-\gamma} \cdot 10 \frac{\frac{-\zeta_{b \rightarrow(i, 0, b)}}{10}}{\left(1-\alpha_{(i, 0, b)}\right)} \\
\leq & E_{\mathrm{c}, b}^{\mathrm{t})} \cdot r_{b \rightarrow(i, 0, b)}^{-\gamma} \cdot 10^{\frac{-\zeta_{b \rightarrow(i, 0, b)}}{10}} \\
\leq & E_{\mathrm{c}, \max }^{(\mathrm{t})} \cdot r_{b \rightarrow(i, 0, b)}^{-\gamma} \cdot 10^{\frac{-\zeta_{b \rightarrow(i, 0, b)}}{10}} .
\end{aligned}
$$

Other cell interference (OCI) chip energy is determined by a summation of the transmission chip energy of adjacent BSs. OCI chip energy at MS $(i, 0, b)$ is given as

$$
\begin{aligned}
& I_{\mathrm{oc},(i, 0, b)=} \sum_{z \neq b} E_{\mathrm{c}, z}^{(\mathrm{t})} \cdot r_{z \rightarrow(i, 0, b)}^{-\gamma} \cdot 10^{\frac{-\zeta_{z \rightarrow(i, 0, b)}}{10}} \\
&=\sum_{z \neq b}\left(\rho E_{\mathrm{c}, \max }^{(\mathrm{t})}+\sum_{(x, 0, z)} \nu_{z \rightarrow(x, 0, z)} E_{\mathrm{c}, z \rightarrow(x, 0, z)}^{(\mathrm{t})}\right) \\
& \qquad r_{z \rightarrow(i, 0, b)}^{-\gamma} \cdot 10^{\frac{-\zeta_{z \rightarrow(i, 0, b)}}{10}} \\
& \leq \sum_{z \neq b} E_{\mathrm{c}, \max }^{(\mathrm{t})} \cdot r_{z \rightarrow(i, 0, b)}^{-\gamma} \cdot 10^{\frac{-\zeta_{z \rightarrow(i, 0, b)}}{10}} \\
&= E_{\mathrm{c}, \max }^{(\mathrm{t})} \cdot \sum_{z \neq b} r_{z \rightarrow(i, 0, b)}^{-\gamma} \cdot 10^{\frac{-\zeta_{z \rightarrow(i, 0, b)}}{10}} .
\end{aligned}
$$

\section{E. Expectations of Interferences and Total Transmission Chip Energy}

From previous sections, the total transmission chip energy of a BS and the interference chip energy at an MS are expressed from the transmission chip energy per MS. To obtain the average system capacity, the expectation values of the interference and the total transmission chip energy must be derived. From (15) and (16), the expectation of the inner/outercell interference at MS $(i, 0, b)$ can be expressed.

Since propagation loss and shadowing are independent of each other, the expectation values from (9) and (11) are used for calculating the expectation of the inner-cell interference chip energy as follows:

$$
\begin{aligned}
E[ & I_{\mathrm{ic},(i, 0, b)]} \\
= & \left(\rho E_{\mathrm{c}, \max }^{(\mathrm{t})}+\sum_{\{(x, 0, b) \mid x \neq i\}} \bar{\nu} \cdot E\left[E_{\mathrm{c}, b \rightarrow(x, 0, b)}^{(\mathrm{t})} \mid \text { active }\right]\right) \\
& \cdot \frac{2}{-\gamma+2}\left(r_{\mathrm{e}}^{-\gamma}-\varepsilon^{-\gamma}\right) \cdot \exp \left\{\frac{\left(\frac{\ln 10}{10} \sigma_{\zeta}\right)^{2}}{2}\right\} \cdot(1-\bar{\alpha}) \\
\leq & E\left[E_{\mathrm{c}, b}^{(\mathrm{t})}\right] \cdot \frac{2}{-\gamma+2}\left(r_{\mathrm{e}}^{-\gamma}-\varepsilon^{-\gamma}\right) \cdot \exp \left\{\frac{\left(\frac{\ln 10}{10} \sigma_{\zeta}\right)^{2}}{2}\right\} \\
\leq & E_{\mathrm{c}, \operatorname{tmax}}^{(\mathrm{t})} \cdot \frac{2}{-\gamma+2}\left(r_{\mathrm{e}}^{-\gamma}-\varepsilon^{-\gamma}\right) \cdot \exp \left\{\frac{\left(\frac{\ln 10}{10} \sigma_{\zeta}\right)^{2}}{2}\right\} .
\end{aligned}
$$

For the outer-cell interference chip energy, the expectation value at $\operatorname{MS}(i, 0, b)$ is derived from (10), (11), and (16) 
as follows:

$$
\begin{aligned}
E & {\left[I_{\mathrm{oc},(i, 0, b)]}\right.} \\
= & \sum_{z \neq b} E\left[E_{\mathrm{c}, z}^{(\mathrm{t})}\right] \cdot E\left[r_{z \rightarrow(i, 0, b)}^{-\gamma}\right] \cdot \exp \left\{\frac{\left(\frac{\ln 10}{10} \sigma_{\zeta}\right)^{2}}{2}\right\} \\
= & \sum_{z \neq b}\left(\rho E_{\mathrm{c}, \max }^{(\mathrm{t})}+\sum_{(x, 0, z)} \bar{\nu} \cdot E\left[E_{\mathrm{c}, z \rightarrow(x, 0, z)}^{(\mathrm{t})} \mid \mathrm{active}\right]\right) \\
& \cdot E\left[r_{z \rightarrow(i, 0, b)}^{-\gamma}\right] \cdot \exp \left\{\frac{\left(\frac{\ln 10}{10} \sigma_{\zeta}\right)^{2}}{2}\right\} \\
\leq & \sum_{z \neq b} E_{\mathrm{c}, \max }^{(\mathrm{t})} \cdot E\left[r_{z \rightarrow(i, 0, b)}^{-\gamma}\right] \cdot \exp \left\{\frac{\left(\frac{\ln 10}{10} \sigma_{\zeta}\right)^{2}}{2}\right\} \\
= & E_{\mathrm{c}, \max }^{(\mathrm{t})} \cdot \exp \left\{\frac{\left(\frac{\ln 10}{10} \sigma_{\zeta}\right)^{2}}{2}\right\} \cdot \sum_{z \neq b} E\left[r_{z \rightarrow(i, 0, b)}^{-\gamma}\right] .
\end{aligned}
$$

The expectation value of the total transmission chip energy from $\mathrm{BS} b$ is derived from the expectation of the transmission chip energy and the activity factor for an MS. Thus, the expectation of total transmission chip energy from $\mathrm{BS} b$ is expressed as

$$
\begin{aligned}
\sum_{(i, 0, b)} & E\left[E_{\mathrm{c}, b \rightarrow(i, 0, b)}^{(\mathrm{t})}\right] \\
& =\sum_{(i, 0, b)}\left(E\left[\nu_{\mathrm{c}, b \rightarrow(i, 0, b)}\right] \cdot E\left[E_{\mathrm{c}, b \rightarrow(i, 0, b)}^{(\mathrm{t})} \mid \text { active }\right]\right)
\end{aligned}
$$

where $\nu_{\mathrm{c}, b \rightarrow(i, 0, b)}$ is the channel activity of the downlink channel from BS $b$ to MS $i$.

We already know a relation of $E\left[E_{\mathrm{c}, b \rightarrow(i, 0, b)}^{(\mathrm{r})} \mid\right.$ active $]=$ $\left(E_{\mathrm{c}} / I_{0}\right)_{\text {target }} \cdot E\left[I_{0,(i, 0, b)}\right]$, and the equations for $E\left[I_{\mathrm{ic},(i, 0, b)}\right]$ and $E\left[I_{\mathrm{oc},(i, 0, b)}\right]$. Therefore, using the previous (17)-(19), the expectation of the total transmission chip energy from $\mathrm{BS} b$ is derived as

$$
\begin{aligned}
\sum_{(i, 0, b)} E & {\left[E_{\mathrm{c}, b \rightarrow(i, 0, b)}^{(\mathrm{t})}\right] } \\
= & M_{b}^{\mathrm{p}} \cdot \bar{\nu} \cdot\left(\frac{E_{\mathrm{c}}}{I_{0}}\right)_{\mathrm{target}} \\
& {\left[\left(\rho E_{\mathrm{c}, \max }^{(\mathrm{t})} \sum_{\{(x, 0, b) \mid x \neq i\}} \bar{\nu} \cdot E\left[E_{\mathrm{c}, b \rightarrow(x, 0, b)}^{(\mathrm{t})} \mid \mathrm{active}\right]\right)\right.} \\
& \cdot \frac{4}{\gamma^{2}-4} \cdot \frac{\left(r_{\mathrm{e}}^{\gamma}-\varepsilon^{\gamma}\right)^{2}}{r_{\mathrm{e}}^{\gamma} \varepsilon^{\gamma}} \cdot \exp \left\{\left(\frac{\ln 10}{10} \sigma_{\zeta}\right)^{2}\right\} \cdot(1-\bar{\alpha}) \\
& +\left\{\sum_{z \neq b}\left(\rho E_{\mathrm{c}, \max }^{(\mathrm{t})}+\sum_{(x, 0, z)} \bar{\nu} \cdot E^{(\mathrm{t})}\left[E_{\mathrm{c}, z \rightarrow(x, 0, z)} \mid \mathrm{active}\right]\right)\right. \\
& \cdot E\left[r_{z \rightarrow(i, 0, b)}^{-\gamma} \cdot \exp \left\{\frac{\left(\frac{\ln 10}{10} \sigma_{\zeta}\right)^{2}}{2}\right\}+N_{0}\right\} \\
& \left.\frac{2}{\gamma+2}\left(r_{\mathrm{e}}^{\gamma}-\varepsilon^{\gamma}\right) \cdot \exp \left\{\frac{\left(\frac{\ln 10}{10} \sigma_{\zeta}\right)^{2}}{2}\right\}\right]
\end{aligned}
$$

The expectation of total transmission chip energy is expressed as the number of downlink channels, the mean channel activity, target received $E_{\mathrm{b}} / I_{0}$ value, and interference values in (20). The detailed derivation is given in Appendix.

\section{F. Power Capacity of OCHM}

Using (20), we can rearrange the inequality $\sum_{(i, 0, b)} E\left[E_{\mathrm{c}, b \rightarrow(i, 0, b)}^{(\mathrm{t})}\right] \leq(1-\rho) E_{\mathrm{c}, \max }^{(\mathrm{t})}$ from (5) and obtain the power capacity in the $b$ th cell $M_{b}^{\mathrm{p}}$ as follows:

$$
\begin{aligned}
M_{b}^{\mathrm{p}} \leq & \left.\frac{(1-\rho) E_{\mathrm{c}, \max }^{(\mathrm{t})}}{\bar{\nu} \cdot\left(\frac{E_{\mathrm{c}}}{I_{0}}\right)_{\mathrm{target}}^{\mathrm{OCHM}}}\right] \\
& {\left[\left(\rho E_{\mathrm{c}, \max }^{(\mathrm{t})}+\sum_{\{(x, 0, b) \mid x \neq i\}} \bar{\nu} \cdot E\left[E_{\mathrm{c}, b \rightarrow(x, 0, b)}^{(\mathrm{t})} \mid \text { active }\right]\right)\right.} \\
& \cdot \frac{4}{\gamma^{2}-4} \cdot \frac{\left(r_{\mathrm{e}}^{\gamma}-\varepsilon^{\gamma}\right)^{2}}{r_{\mathrm{e}}^{\gamma} \varepsilon^{\gamma}} \cdot \exp \left\{\left(\frac{\ln 10}{10} \sigma_{\zeta}\right)^{2}\right\} \cdot(1-\bar{\alpha}) \\
+ & \left\{\sum_{z \neq b}\left(\rho E_{\mathrm{c}, \max }^{(\mathrm{t})}+\sum_{(x, 0, z)} \bar{\nu} \cdot E\left[E_{\mathrm{c}, z \rightarrow(x, 0, z)}^{(\mathrm{t})} \mid \mathrm{active}\right]\right)\right. \\
& \left.\cdot E^{2}\left[r_{z \rightarrow(i, 0, b)}^{-\gamma}\right] \cdot \exp \left\{\frac{\left(\frac{\ln 10}{10} \sigma_{\zeta}\right)^{2}}{2}\right\}+N_{0}\right\} \\
& \left.\frac{2}{\gamma+2}\left(r_{\mathrm{e}}^{\gamma}-\varepsilon^{\gamma}\right) \cdot \exp \left\{\frac{\left(\frac{\ln 10}{10} \sigma_{\zeta}\right)^{2}}{2}\right\}\right]
\end{aligned}
$$

Equation (21) shows the system capacity based on a powerlimited situation. $M_{b}^{\mathrm{p}}$ is bounded by the terms proportional to the maximum transmission chip energy of the $\mathrm{BS}, E_{\mathrm{c}, \max }^{(\mathrm{t})}$, and inversely proportional to the mean channel activity $\bar{\nu}$ and the received target signal-to-interference-plus-noise ratio (SINR) $\left(E_{\mathrm{c}} / I_{0}\right)_{\text {target }}^{\text {OCHM }}$.

We define three different cases of outer-cell interference. The first one is the maximum outer-cell interference case, which is the worst case. Generally, the capacity or the number of admissible downlink channels is obtained in this case, and the maximum transmission power is also adjusted in this case. The second case is that all adjacent cells have no downlink channels, except common control channels such as a pilot control channel. The third case is that there are no adjacent cells with the same serving frequency band. Thus, the frequency reuse factor is far less than 1 .

When the BSs of all adjacent cells transmit at the allowable maximum transmission power, we can derive the probabilistically worst system capacity. The probabilistically worst case is not the worst case in reality. The real worst case here means the case where all MSs served by a BS are located around the cell boundary covered by the BS. That is, the real worst case occurs when the transmission power per channel is maximally allocated. However, the probabilistically worst case occurs when all MSs served by a BS are uniformly located 
within the cell covered by the BS, and the BSs of all adjacent cells transmit at the allowable maximum transmission power. If outer-cell interference is a maximum, (21) is expressed as

$$
\begin{aligned}
& M_{b}^{\mathrm{p}, \max \text { OCI }} \leq \frac{(1-\rho) E_{\mathrm{c}, \max }^{(\mathrm{t})}}{\bar{\nu} \cdot\left(\frac{E_{\mathrm{c}}}{I_{0}}\right)_{\mathrm{target}}^{\mathrm{tCHM}}} / \\
& {\left[\left(\rho E_{\mathrm{c}, \max }^{(\mathrm{t})}+\sum_{\{(x, 0, b) \mid x \neq i\}} \bar{\nu} \cdot E\left[E_{\mathrm{c}, b \rightarrow(x, 0, b)}^{(\mathrm{t})} \mid \operatorname{active}\right]\right)\right.} \\
& \cdot \frac{4}{\gamma^{2}-4} \cdot \frac{\left(r_{\mathrm{e}}^{\gamma}-\varepsilon^{\gamma}\right)^{2}}{r_{\mathrm{e}}^{\gamma} \varepsilon^{\gamma}} \cdot \exp \left\{\left(\frac{\ln 10}{10} \sigma_{\zeta}\right)^{2}\right\} \cdot(1-\bar{\alpha}) \\
& +\left\{\sum_{z \neq b} E_{\mathrm{c}, \max }^{(\mathrm{t})} \cdot E\left[r_{z \rightarrow(i, 0, b)}^{-\gamma}\right] \cdot \exp \left\{\frac{\left(\frac{\ln 10}{10} \sigma_{\zeta}\right)^{2}}{2}\right\}+N_{0}\right\} \\
& \left.\quad \cdot \frac{2}{\gamma+2}\left(r_{\mathrm{e}}^{\gamma}-\varepsilon^{\gamma}\right) \cdot \exp \left\{\frac{\left(\frac{\ln 10}{10} \sigma_{\zeta}\right)^{2}}{2}\right\}\right]
\end{aligned}
$$

where $M_{b}^{\mathrm{p}, \text { max OCI }}$ is the power capacity when OCI has a maximum value.

When BSs of all adjacent cells transmit at the minimum transmission power, such as the pilot channel power, we can derive the probabilistically best system capacity. The probabilistically best case is not the best case in reality. The real best case means the case where all MSs served by a BS are located at the nearest position to the BS and the transmission power per channel is minimally allocated. However, the probabilistically best case occurs when all MSs served by a BS are uniformly located within the cell covered by the BS, and BSs of all adjacent cells transmit at the minimum transmission power, such as the pilot channel power. The number of downlink channels in a cell is bounded, as in the minimum OCI case

$$
\begin{aligned}
& M_{b}^{\mathrm{p}, \min \text { OCI }} \leq \frac{(1-\rho) E_{\mathrm{c}, \text { max }}^{\mathrm{t})}}{\bar{\nu} \cdot\left(\frac{E_{\mathrm{c}}}{I_{\mathrm{o}}}\right)_{\mathrm{target}}^{\mathrm{OCHM}}} / \\
& {\left[\left(\rho E_{\mathrm{c}, \text { max }}^{(\mathrm{t})}+\sum_{\{(x, 0, b) \mid x \neq i\}} \bar{\nu} \cdot E\left[E_{\mathrm{c}, b \rightarrow(x, 0, b)}^{(\mathrm{t})} \mid \text { active }\right]\right)\right.} \\
& \quad \frac{4}{\gamma^{2}-4} \cdot \frac{\left(r_{\mathrm{e}}^{\gamma}-\varepsilon^{\gamma}\right)^{2}}{r_{\mathrm{e}}^{\gamma} \varepsilon^{\gamma}} \cdot \exp \left\{\left(\frac{\ln 10}{10} \sigma_{\zeta}\right)^{2}\right\} \cdot(1-\bar{\alpha}) \\
& +\left\{\sum_{z \neq b} \rho E_{\mathrm{c}, \max }^{(\mathrm{t})} \cdot E\left[r_{z \rightarrow(i, 0, b)}^{-\gamma}\right] \cdot \exp \left\{\frac{\left(\frac{\ln 10}{10} \sigma_{\zeta}\right)^{2}}{2}\right\}+N_{0}\right\} \\
& \left.\quad \cdot \frac{2}{\gamma+2}\left(r_{\mathrm{e}}^{\gamma}-\varepsilon^{\gamma}\right) \cdot \exp \left\{\frac{\left(\frac{\ln 10}{10} \sigma_{\zeta}\right)^{2}}{2}\right\}\right]
\end{aligned}
$$

where $M_{b}^{\mathrm{p}, \text { min OCI }}$ is the power capacity when OCI has a minimum value.

\section{G. Power Capacity in Sectorized Cell Environments}

Even if omnicells are sectorized, the total transmission chip energy from BS $b$ is assumed to be identical to (20). A proportion of the total transmission power from sector antennas of adjacent BSs affects the BS $b$ as OCI. The interference power is determined by the sector antenna characteristics, such as the main lobe width and the side lobe attenuation. As an ideal approximation, we assume that an antenna with a main lobe width of $\theta$ directly reduces the average OCI power by a factor of $\theta / 360$. Thus, when a cell is sectorized with a transmission antenna of main lobe width $\theta(0 \leq \theta \leq 360)$, the power capacities in a sectorized cell are modified from (22) and (23) and are expressed as

$$
\begin{aligned}
& M_{b}^{\mathrm{p}, \max \text { OCI }, \theta} \leq \frac{(1-\rho) E_{\mathrm{c}, \max }^{(\mathrm{t})}}{\bar{\nu} \cdot\left(\frac{E_{\mathrm{c}}}{I_{0}}\right)_{\mathrm{target}}^{\mathrm{OCHM}}} / \\
& {\left[\left(\rho E_{\mathrm{c}, \max }^{(\mathrm{t})}+\sum_{\{(x, 0, b) \mid x \neq i\}} \bar{\nu} \cdot E\left[E_{\mathrm{c}, b \rightarrow(x, 0, b)}^{(\mathrm{t})} \mid \operatorname{active}\right]\right)\right.} \\
& \cdot \frac{4}{\gamma^{2}-4} \cdot \frac{\left(r_{\mathrm{e}}^{\gamma}-\varepsilon^{\gamma}\right)^{2}}{r_{\mathrm{e}}^{\gamma} \varepsilon^{\gamma}} \cdot \exp \left\{\left(\frac{\ln 10}{10} \sigma_{\zeta}\right)^{2}\right\} \cdot(1-\bar{\alpha}) \\
& +\left\{\sum_{z \neq b} E_{\mathrm{c}, \max }^{(\mathrm{t})} \cdot E\left[r_{z \rightarrow(i, 0, b)}^{-\gamma}\right]\right. \\
& \left.\cdot \exp \left\{\frac{\left(\frac{\ln 10}{10} \sigma_{\zeta}\right)^{2}}{2}\right\} \cdot \frac{\theta}{360}+N_{0}\right\} \\
& \left.\quad \cdot \frac{2}{\gamma+2}\left(r_{\mathrm{e}}^{\gamma}-\varepsilon^{\gamma}\right) \cdot \exp \left\{\frac{\left(\frac{\ln 10}{10} \sigma_{\zeta}\right)^{2}}{2}\right\}\right]
\end{aligned}
$$

$$
\begin{aligned}
& M_{b}^{\mathrm{p}, \min \text { OCI }, \theta} \leq \frac{(1-\rho) E_{\mathrm{c}, \max }^{(\mathrm{t})}}{\bar{\nu} \cdot\left(\frac{E_{\mathrm{c}}}{I_{0}}\right)_{\text {target }}^{\mathrm{OCHM}}} / \\
& {\left[\left(\rho E_{\mathrm{c}, \max }^{(\mathrm{t})}+\sum_{\{(x, 0, b) \mid x \neq i\}} \bar{\nu} \cdot E\left[E_{\mathrm{c}, b \rightarrow(x, 0, b)}^{(\mathrm{t})} \mid \text { active }\right]\right)\right.} \\
& \cdot \frac{4}{\gamma^{2}-4} \cdot \frac{\left(r_{\mathrm{e}}^{\gamma}-\varepsilon^{\gamma}\right)^{2}}{r_{\mathrm{e}}^{\gamma} \varepsilon^{\gamma}} \cdot \exp \left\{\left(\frac{\ln 10}{10} \sigma_{\zeta}\right)^{2}\right\} \cdot(1-\bar{\alpha}) \\
& +\left\{\sum_{z \neq b} \rho E_{\mathrm{c}, \max }^{(\mathrm{t})} \cdot E\left[r_{z \rightarrow(i, 0, b)}^{-\gamma}\right]\right. \\
& \left.\cdot \exp \left\{\frac{\left(\frac{\ln 10}{10} \sigma_{\zeta}\right)^{2}}{2}\right\} \cdot \frac{\theta}{360}+N_{0}\right\} \\
& \left.\quad \frac{2}{\gamma+2}\left(r_{\mathrm{e}}^{\gamma}-\varepsilon^{\gamma}\right) \cdot \exp \left\{\frac{\left(\frac{\ln 10}{10} \sigma_{\zeta}\right)^{2}}{2}\right\}\right] .
\end{aligned}
$$


TABLE I

ASSUMPTIONS FOR NUMERICAL EXAMPLES

\begin{tabular}{|c|r||c|r|}
\hline Parameter & Value & Parameter & Value \\
\hline \hline$r_{e}$ & $1000[\mathrm{~m}]$ & $\varepsilon$ & $10[\mathrm{~m}]$ \\
\hline$\gamma$ & 4 & $\sigma_{\zeta}$ & $8[\mathrm{~dB}]$ \\
\hline$N_{O C}$ & 64 & $R^{F E C}$ & $1 / 4$ \\
\hline $\bar{\alpha}$ & 1.0 & $N_{0}$ & $-174[\mathrm{dBm}]$ \\
\hline$E_{c, \text { max }}^{(t)}$ & $\frac{15[W]}{1.2288[M c p s]}$ & $\Delta\left(\frac{E_{b}}{I_{0}}\right)_{\text {target }}$ & $0.5[\mathrm{~dB}]$ \\
\hline
\end{tabular}

\section{OVERALl SYSTEM CAPACITY AND NUMERICAL EXAMPLES OF OCHM}

\section{A. Overall System Capacity in OCHM}

In previous sections, we evaluated the code capacity in (4) and the power capacity in (21). Therefore, the overall system capacity $M_{b}^{\max }$ considering both code and power limitations can be expressed as

$$
M_{b}^{\max }=\min \left(M_{b}^{\mathrm{c}}, M_{b}^{\mathrm{p}}\right)
$$

where $M_{b}^{\mathrm{c}}$ and $M_{b}^{\mathrm{p}}$ represent the code and power capacities determined by (4) and (21), respectively.

In OCHM, both power capacity and code capacity vary depending on the perforation probability, the mean channel activity, and the number of codewords. The overall capacity determined by $M_{b}^{\mathrm{p}}$ can be called a power limited state. On the other hand, the overall capacity equal to $M_{b}^{c}$ can be called a code-limited or perforation-limited state since the code capacity is limited by the allowable perforation probability in OCHM.

\section{B. Numerical Examples for the Overall System Capacity}

The physical layer parameter $\left(E_{\mathrm{b}} / I_{0}\right)_{\text {target }}^{\mathrm{OCHM}}$ has a typical range from 0 to $7 \mathrm{~dB}$, and the channel code rate is fixed at $1 / 4$. The total transmission chip energy $E_{\mathrm{c}, \max }^{(\mathrm{t})}$ is calculated by multiplication of the maximum transmission power of a BS and the chip duration. A typical value of the maximum transmission power is $15 \mathrm{~W}$, and the chip duration is $1 / 1.2288[\mathrm{MHz}]$ in cdma2000 [3]. We consider two typical channel-activity values of 0.1 and 0.2 since OCHM targets packet services. In this example, the tolerable value of $\Delta\left(E_{\mathrm{b}} / I_{0}\right)_{\text {target }}$, which is the difference between the target $E_{\mathrm{b}} / I_{0}$ values of OCDM and OCHM, is set at $0.5 \mathrm{~dB}$ with an FER value of 0.01 . Thus, the allowable perforation probability is 0.25 , and the required $\left(E_{\mathrm{b}} / I_{0}\right)_{\text {target }}^{\mathrm{OCHM}}$ value is approximately $2.0 \mathrm{~dB}$ with TC from Fig. 4. The detailed environment is shown in Table I.

Fig. 6 shows the code and power capacities versus the $\left(E_{\mathrm{b}} / I_{0}\right)_{\text {target }}^{\text {OCHM }}$ value. A horizontal solid line without marks corresponds to the code capacity of OCDM, which is equal to 64 codewords, and six parallel solid lines with marks correspond to the six code capacities of OCHM. For a mean channel activity of $\bar{\nu}=0.1$, the six perforation probabilities $p_{\mathrm{p}, \mathrm{OC}}=0.30, \quad p_{\mathrm{p}, \mathrm{OC}}=0.25, \quad p_{\mathrm{p}, \mathrm{OC}}=0.20, \quad p_{\mathrm{p}, \mathrm{OC}}=0.15$, $p_{\mathrm{p}, \mathrm{OC}}=0.10$, and $p_{\mathrm{p}, \mathrm{OC}}=0.05$ correspond to code capacities $M_{b}^{\mathrm{c}}=458(>64), M_{b}^{\mathrm{c}}=370(>64), M_{b}^{\mathrm{c}}=287(>64)$, $M_{b}^{c}=209(>64), M_{b}^{c}=136(>64)$, and $M_{b}^{c}=67(>64)$, respectively, which are obtained from Fig. 3 . The five curves with marks represent the OCHM power capacities calculated from (21).

Fig. 6(a) shows the code and power capacities of OCHM in an omnicell environment with a maximum value of OCI. If we assume that the system can support a perforation probability of 0.25 and the proportion of the maximum downlink transmission power for the downlink common control channel $\rho$ is 0.20 , the code capacity is 370 and the power capacity follows the "+" marked curve. In this case, the OCHM system with $p_{\mathrm{p}, \mathrm{OC}}=0.25$ is not code (or perforation) limited but is power limited because a code capacity of 370 is always larger than the power capacity curve. Thus, the overall system capacity of OCHM follows the "+" marked power capacity curve in the whole range of $\left(E_{\mathrm{b}} / I_{0}\right)_{\text {target }}^{\mathrm{OCHM}}$ values. The overall system capacity of OCHM is approximately 180 for an $\left(E_{\mathrm{b}} / I_{0}\right)_{\mathrm{target}}^{\mathrm{OCHM}}$ value of $2.0 \mathrm{~dB}$. For other $\rho$ values, the overall system capacities of OCHM are always power limited in this environment.

Fig. 6(b) illustrates the code and power capacities in an omnicell environment with a minimum OCI value. In this case, the system is code (or perforation) limited for a small value of $\left(E_{\mathrm{b}} / I_{0}\right)_{\text {target }}^{\mathrm{OCHM}}$ since the power capacities increase regardless of the same code capacities. Crossover points depend on the power proportion of the downlink common channels $\rho$. Since OCI has a minimum value, $\rho$ affects the power capacity more than for the maximum OCI case. If $\rho=0.20$ and $\left(E_{\mathrm{b}} / I_{0}\right)_{\text {target }}^{\mathrm{OCHM}} \leq$ $5.5 \mathrm{~dB}$, the overall system capacity of OCHM is determined by the code capacity. However, if $\left(E_{\mathrm{b}} / I_{0}\right)_{\mathrm{target}}^{\mathrm{OCHM}}>5.5 \mathrm{~dB}$, the overall system capacity follows the power capacity curve of $\rho=0.20$. Therefore, the overall system capacity can be 370 at an $\left(E_{\mathrm{b}} / I_{0}\right)_{\text {target }}^{\mathrm{OCHM}}$ value of $2.0 \mathrm{~dB}$. Compared to the maximum OCI case, an increase in the power capacity makes it possible for the system to utilize the whole code capacity of 370 .

Fig. 7 shows the code and power capacities with a mean channel activity of 0.2. Compared to the code and power capacities in Fig. 6, the higher channel activity of 0.2 yields a smaller code capacity due to a small statistical multiplexing gain, and a smaller power capacity due to a large average interference. The code capacities for $p_{\mathrm{p}, \mathrm{OC}}=0.30, p_{\mathrm{p}, \mathrm{OC}}=0.25$, $p_{\mathrm{p}, \mathrm{OC}}=0.20, p_{\mathrm{p}, \mathrm{OC}}=0.15, p_{\mathrm{p}, \mathrm{OC}}=0.10$, and $p_{\mathrm{p}, \mathrm{OC}}=0.05$ are $M_{b}^{c}=230(>64), M_{b}^{c}=185(>64), M_{b}^{c}=144(>64)$, $M_{b}^{\mathrm{c}}=105(>64), M_{b}^{\mathrm{c}}=69(>64)$, and $M_{b}^{\mathrm{c}}=34(<64)$, respectively. The power capacity curve values decrease to approximately half of the curve values for a mean channel activity of 0.1. In Fig. 7(a) and (b), the overall system capacities are limited by power or code (or perforation). The trend is similar to that shown in Fig. 6(a) and (b). Only the $y$-axis is scaled down since both code and power capacities decrease. Therefore, the overall system capacities at an $\left(E_{\mathrm{b}} / I_{0}\right)_{\text {target }}^{\mathrm{OCHM}}$ value of $2.0 \mathrm{~dB}$ decrease to 80 and 185 for $\bar{\nu}=0.2$ compared to 180 and 370 for $\bar{\nu}=0.1$, respectively. However, these values are still larger than the number of codewords equal to 64 .

Fig. 8 shows the overall system capacity of OCHM and OCDM in an omnicell environment. The OCI values of the $x$-axis are normalized by the maximum OCI value. The four solid lines represent the overall system capacities of OCHM with $\left(E_{\mathrm{b}} / I_{0}\right)_{\text {target }}^{\text {OCHM }}$ values of $2.0,3.0,4.0$, and $5.0 \mathrm{~dB}$. In OCDM, the power capacity is also calculated by (21) replacing 


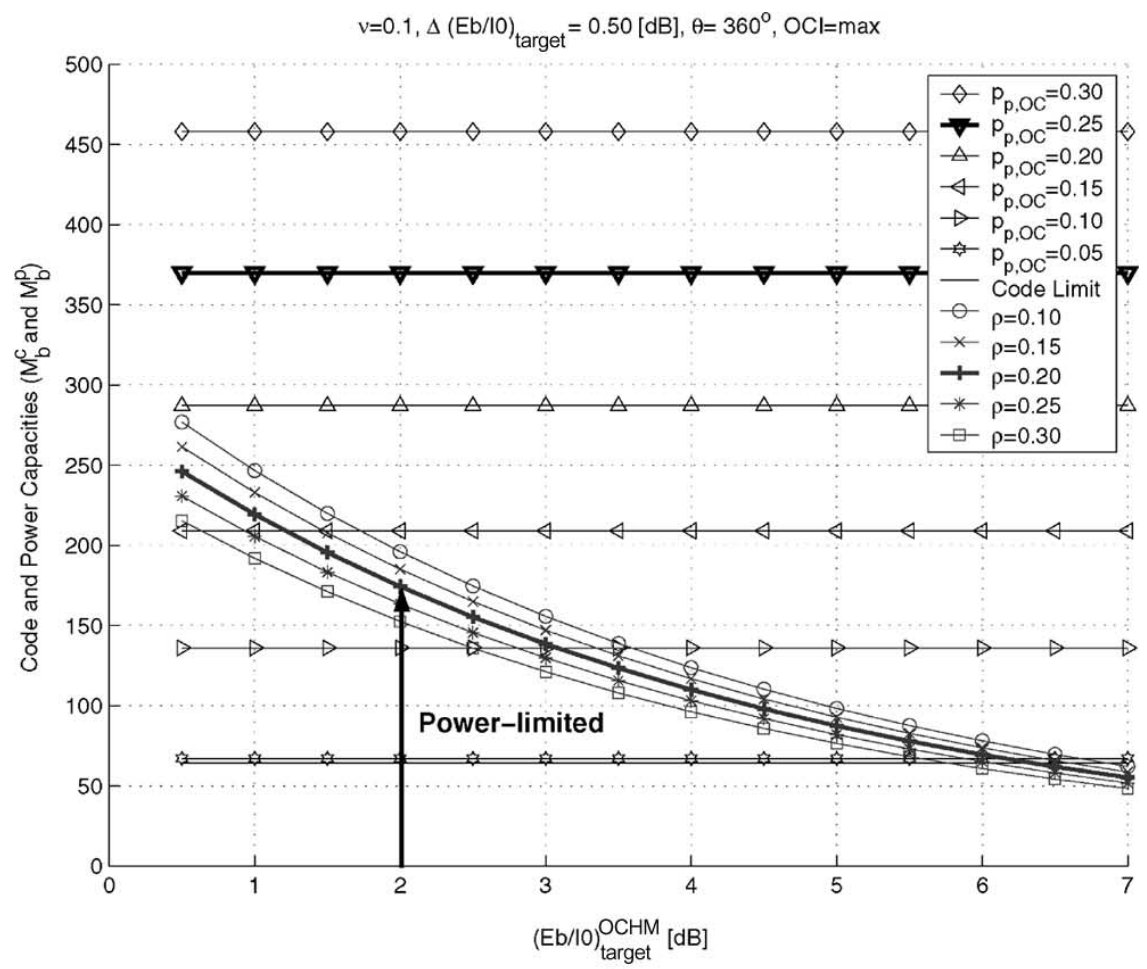

(a)

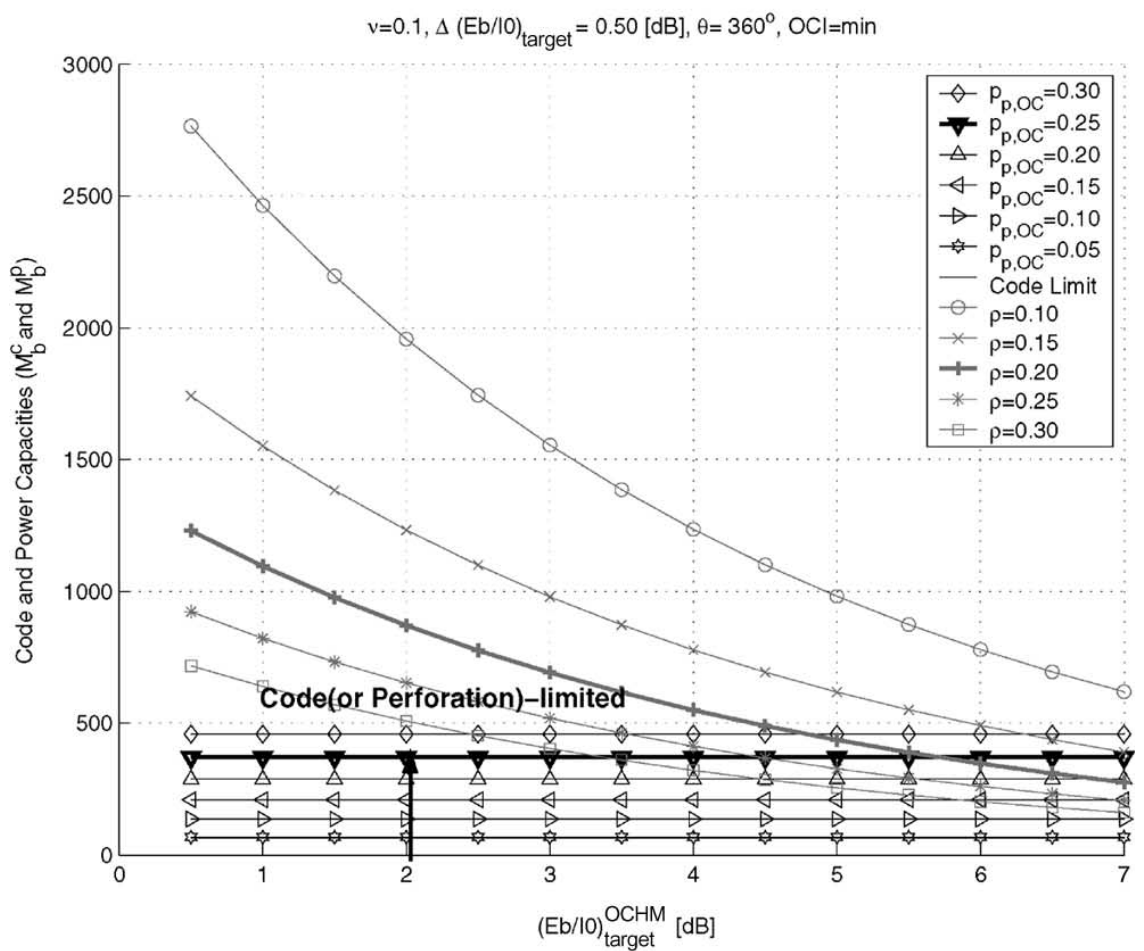

(b)

Fig. 6. Code and power capacities with a mean channel-activity value of 0.1 in an omnicell environment. (a) $\mathrm{OCI}=\max$. (b) $\mathrm{OCI}=\min$.

$\left(E_{\mathrm{b}} / I_{0}\right)_{\text {target }}^{\text {OCHM }}$ with $\left(E_{\mathrm{b}} / I_{0}\right)_{\text {target }}^{\mathrm{OCDM}}$, which is $\left(E_{\mathrm{b}} / I_{0}\right)_{\text {target }}^{\text {OCHM }}$ $\Delta\left(E_{\mathrm{b}} / I_{0}\right)_{\text {target }}$. The four dashed lines represent the overall system capacities of OCDM with $\left(E_{\mathrm{b}} / I_{0}\right)_{\text {target }}^{\text {OCDM }}$ values of 1.5 , $2.5,3.5$, and $4.5 \mathrm{~dB}$. With a mean channel-activity value of 0.1 shown in Fig. 8(a), the OCDM system is always limited by the number of codewords, and the overall system capacity is fixed at an OCDM code capacity of 64 . However, OCHM achieves much larger capacities than OCDM in the entire range because OCHM with a small channel activity yields an increase in both the power capacity and the code capacity due to a statistical multiplexing gain. In Fig. 8(b), OCHM also shows better overall system capacity with a small range of normalized OCI values and a small $\left(E_{\mathrm{b}} / I_{0}\right)_{\text {target }}^{\text {OCHM }}$ value. For normalized OCI values larger than 0.85 and 0.70 with $\left(E_{\mathrm{b}} / I_{0}\right)_{\text {target }}^{\mathrm{OCHM}}=4.0 \mathrm{~dB}$ 


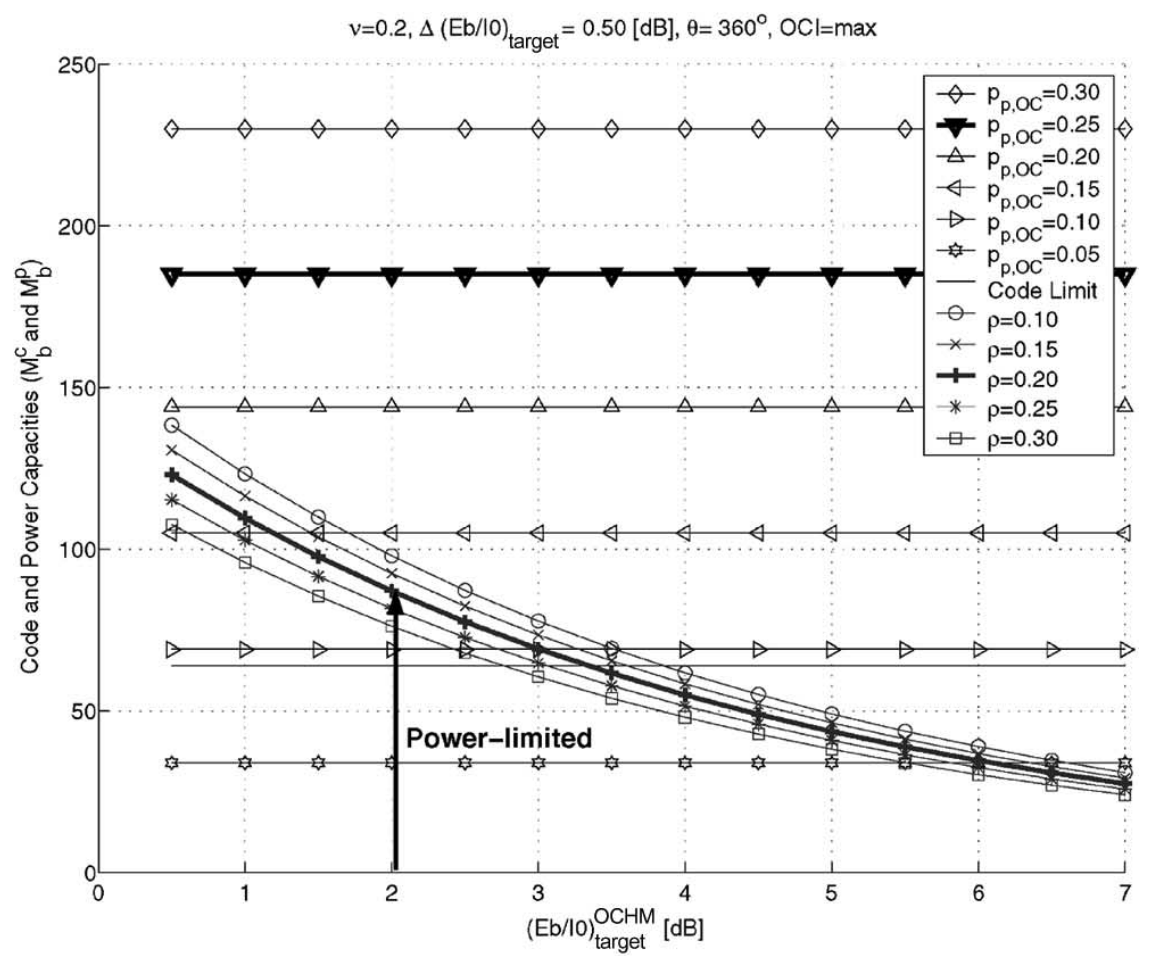

(a)

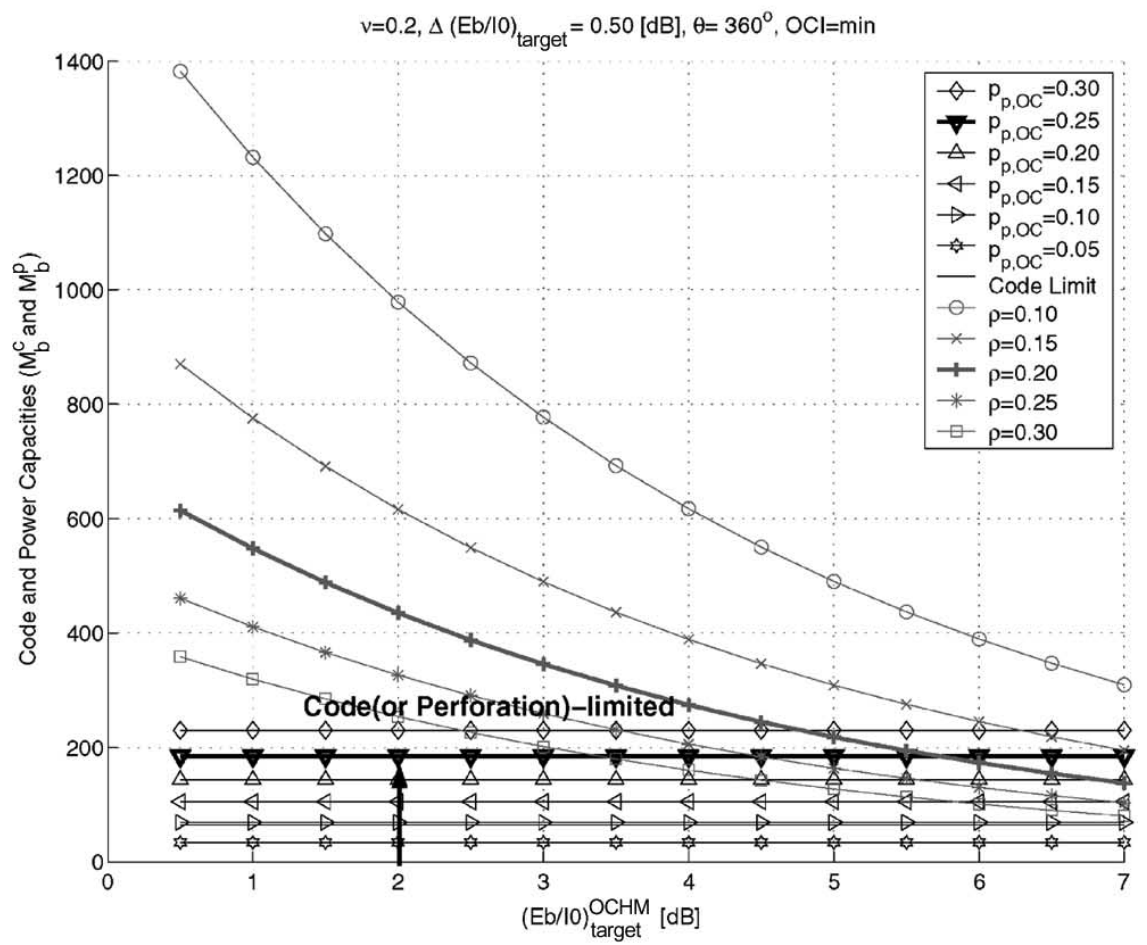

(b)

Fig. 7. Code and power capacities with a mean channel-activity value of 0.2 in an omnicell environment. (a) OCI $=$ max. (b) $\mathrm{OCI}=\min$.

and $\left(E_{\mathrm{b}} / I_{0}\right)_{\text {target }}^{\text {OCHM }}=5.0 \mathrm{~dB}$, respectively, OCHM performs worse than OCDM because both OCHM and OCDM are limited by the power capacity, which is smaller than the number of codewords, and the required $E_{\mathrm{b}} / I_{0}$ value of OCHM is larger than that for OCDM.

Figs. 9 and 10 show the code and power capacities in a three-sectorized cell environment. Compared to Figs. 6 and 7, the power capacities increase because interference from/to other cells in the three-sectorized cell environment is smaller than in the omnidirectional cell environment. However, the code capacities in Figs. 9 and 10 are identical to the values in Figs. 6 and 7, respectively. For the maximum OCI values shown in Figs. 9(a) and 10(a), the overall system capacity is code (or perforation) limited or power limited, depending on 


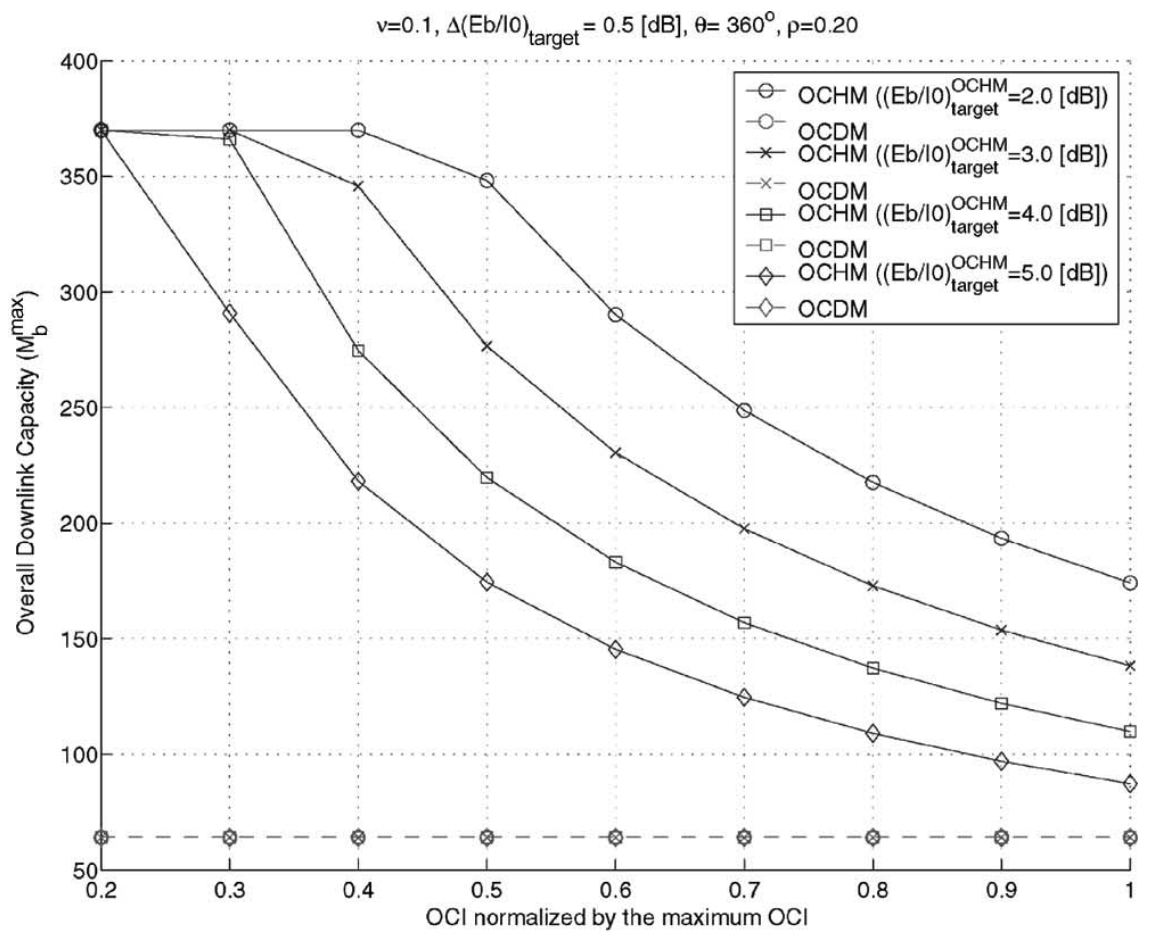

(a)

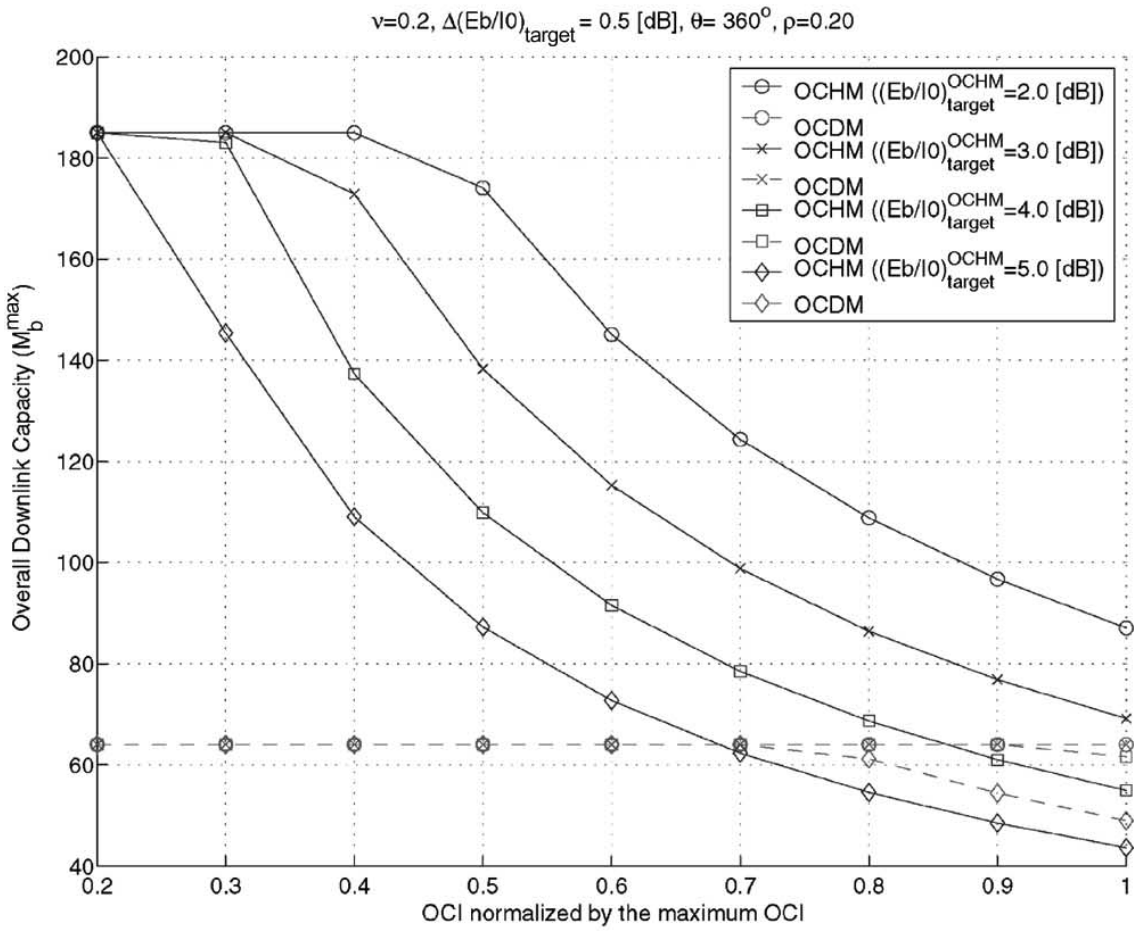

(b)

Fig. 8. Overall system capacity of OCDM and OCHM in an omnicell environment. (a) OCI $=$ max. (b) OCI $=\min$.

$\left(E_{\mathrm{b}} / I_{0}\right)_{\text {target }}^{\text {OCHM }}$ values, while the systems are always power limited in the omnicell environment with a maximum OCI value. The sectorized cell fully achieves the code capacities of 370 and 185 at an $\left(E_{\mathrm{b}} / I_{0}\right)_{\text {target }}^{\text {OCHM }}$ value of $2.0 \mathrm{~dB}$ even with a maximum OCI value, as shown in Figs. 9(a) and 10(a), respectively. For the minimum OCI value shown in Figs. 9(b) and 10(b), the overall system capacities are always code (or perforation) limited under the given conditions. Therefore, where
$\left(E_{\mathrm{b}} / I_{0}\right)_{\text {target }}^{\mathrm{OCHM}}=2.0 \mathrm{~dB}$ and $\rho=0.20$, the overall system capacities are still 370 and 185 , as in the omnicell environment shown in Figs. 6(b) and 7(b), respectively.

Fig. 11 shows the overall system capacity of OCHM and OCDM in a three-sectorized cell environment. The OCHM system is code (or perforation) limited in a range of normalized OCI values larger than 0.8 or 0.7 with $\left(E_{\mathrm{b}} / I_{0}\right)_{\text {target }}^{\mathrm{OCHM}}=4.0 \mathrm{~dB}$ and $\left(E_{\mathrm{b}} / I_{0}\right)_{\text {target }}^{\mathrm{OCHM}}=5.0 \mathrm{~dB}$, respectively. Compared to Fig. 8, 


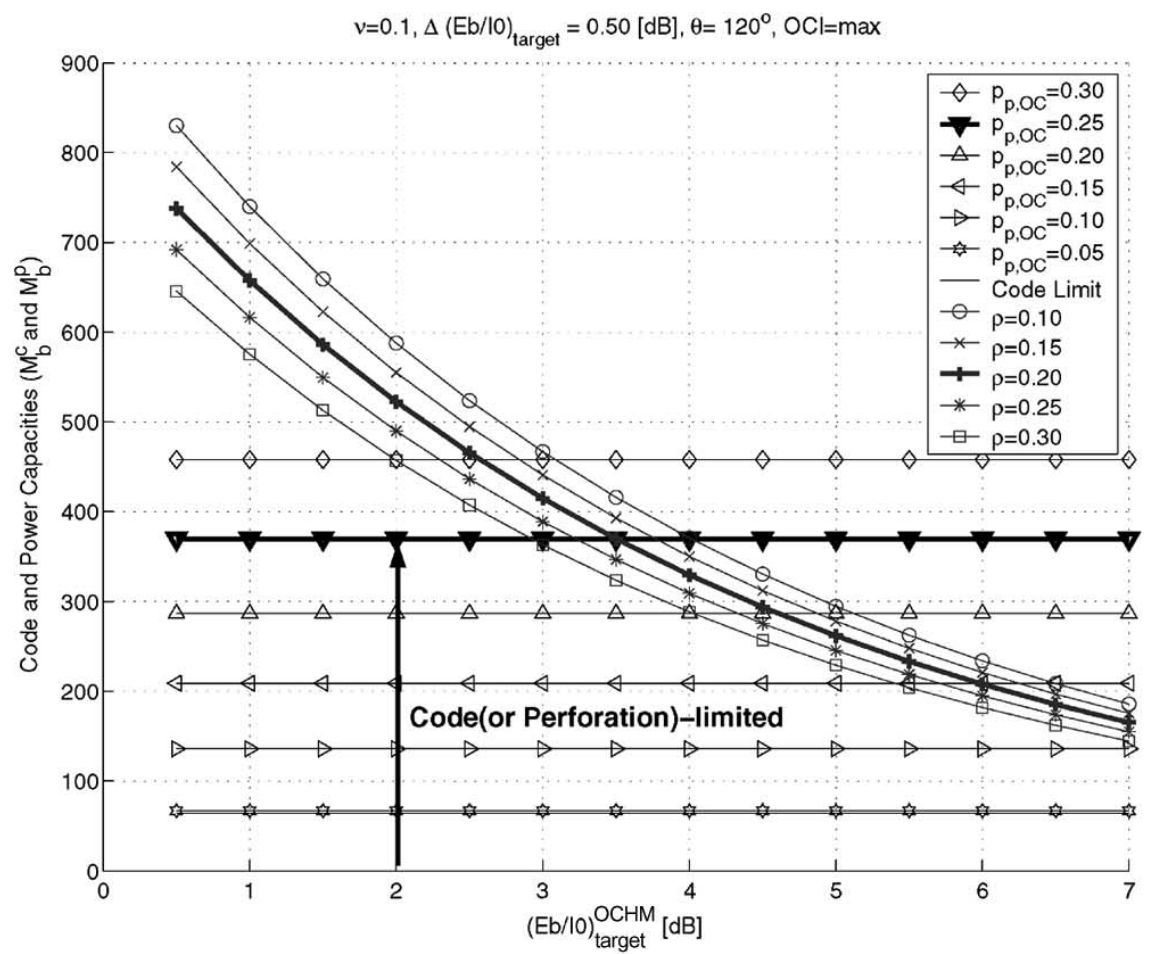

(a)

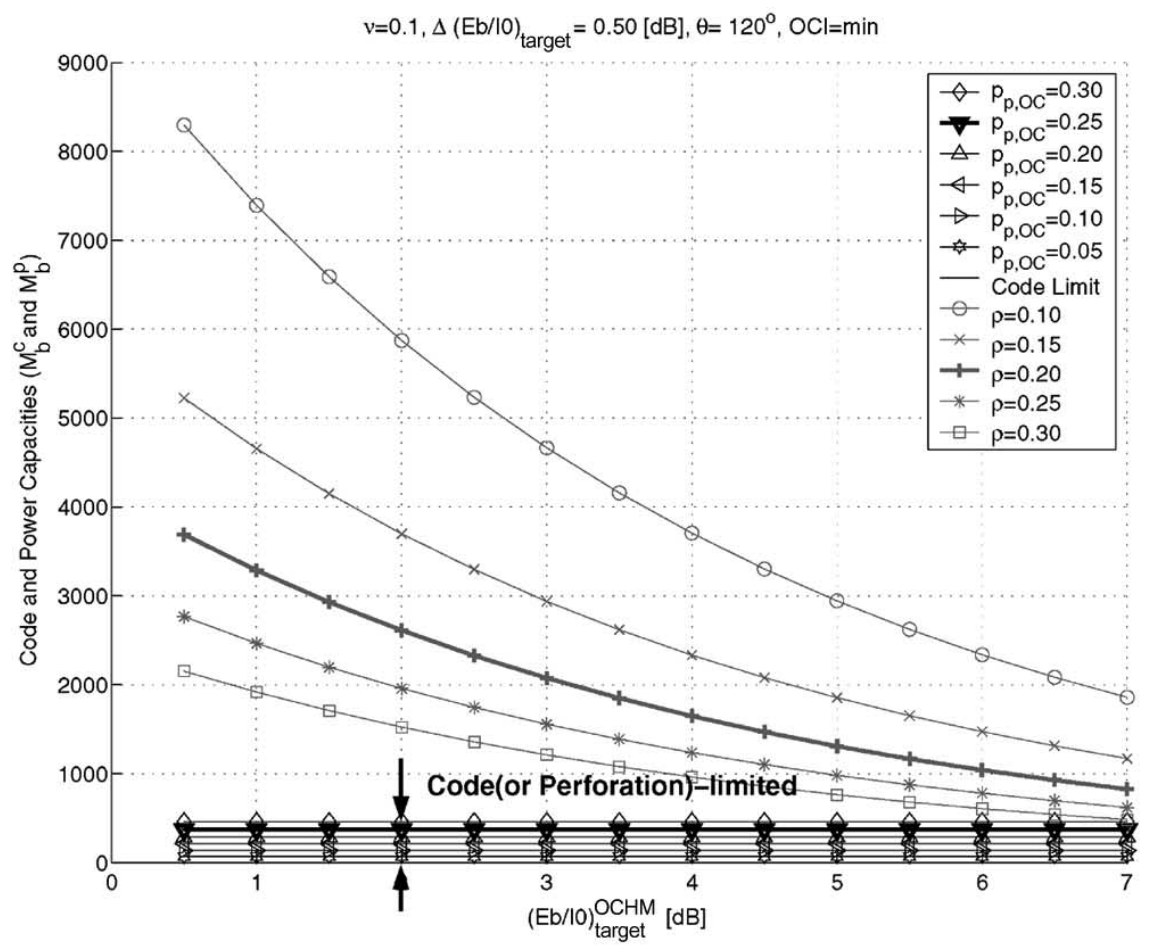

(b)

Fig. 9. Code and power capacities with a mean channel-activity value of 0.1 in a three-sectorized cell environment. (a) OCI $=$ max. (b) OCI $=$ min.

the sectorized cell yields a larger overall system capacity in OCHM while the OCDM system is still limited by the number of codewords. OCHM always performs better than OCDM, even where $\bar{\nu}=0.2$. An increase in the power capacity from the sectorized main lobe width is not effective in OCDM in relatively low-channel-activity environments. In OCHM, an increase in the power capacity from the sectorized main lobe width contributes to an increase in the overall system capacities in large normalized OCI ranges in Fig. 8.

\section{CONCLUSION}

For evaluating the overall system capacity of our previously proposed OCHM, the power capacity of OCHM is analyzed 


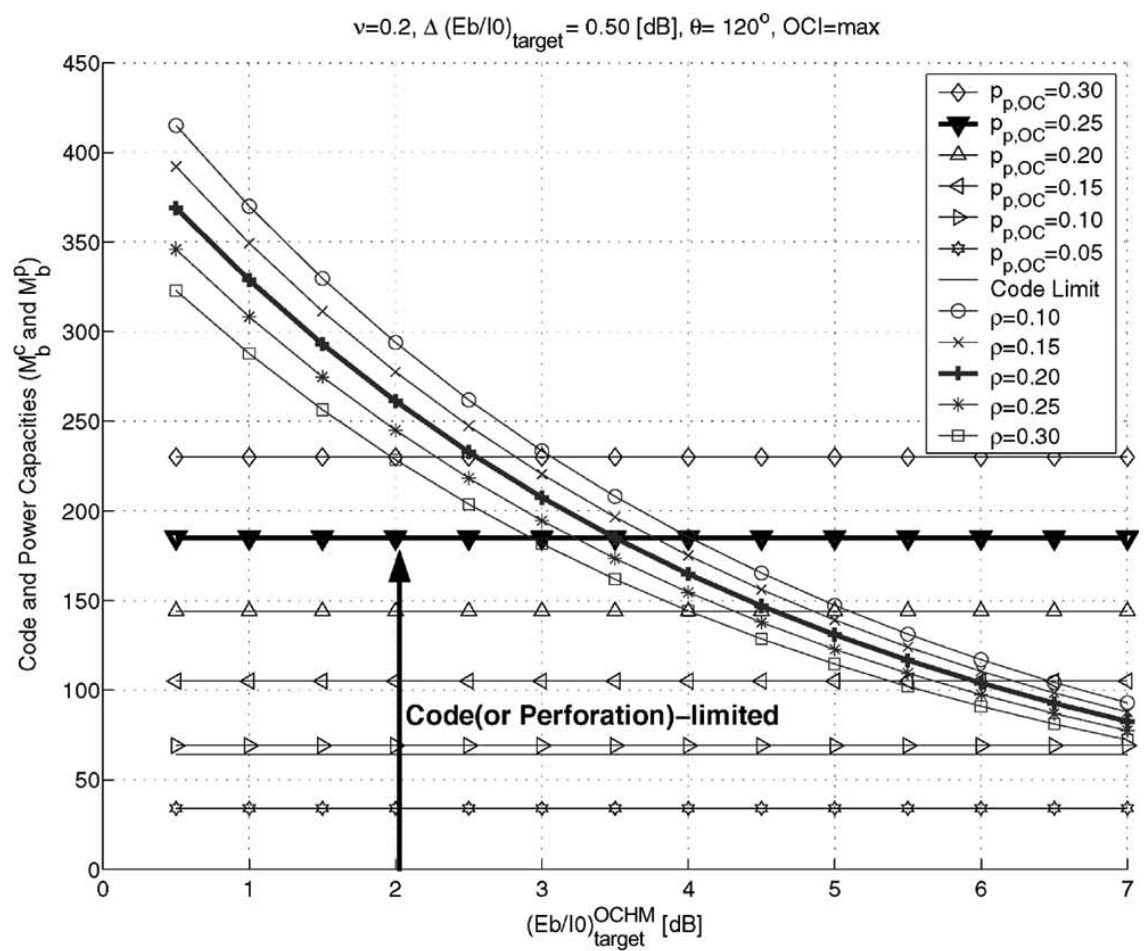

(a)

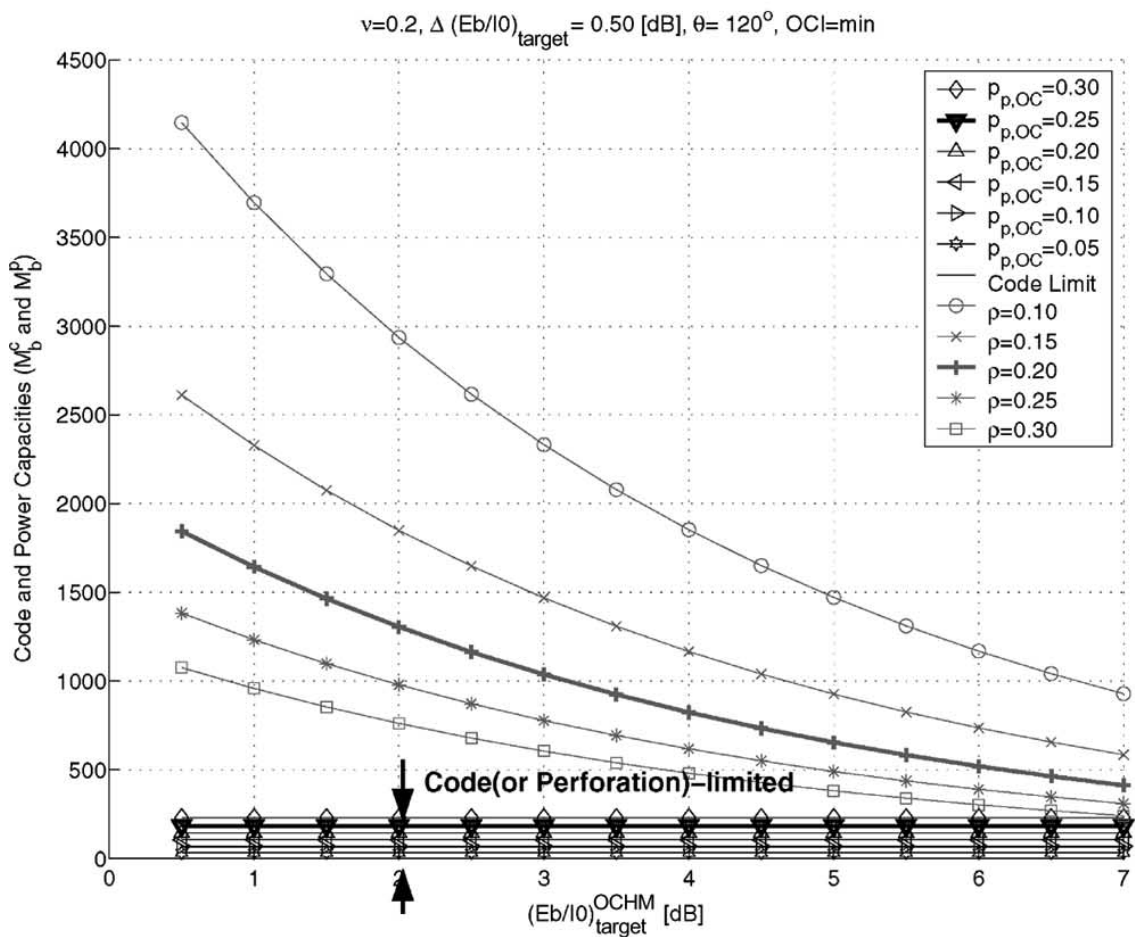

(b)

Fig. 10. Code and power capacities with a mean channel-activity value of 0.2 in a three-sectorized cell environment. (a) OCI $=$ max. (b) OCI $=$ min.

under assumptions of a one-tier hexagonal cell structure and uniformly distributed users. Even if the power capacity is mathematically derived in a simplified environment, we can estimate the effect of various downlink system parameters: mean channel activity, transmission power proportion of the downlink common channels, OCI, and the required (or target) $E_{\mathrm{b}} / I_{0}$ value. From this power capacity and code capacity previously analyzed, the overall system capacity, which is determined from the minimum value of the code and power capacities, can be obtained in the CDMA downlink. In numerical examples, the code, power, and overall capacities are calculated and compared to the values of the conventional CDMA (OCDM). The proposed OCHM system can accommodate more downlink channels than OCDM because 


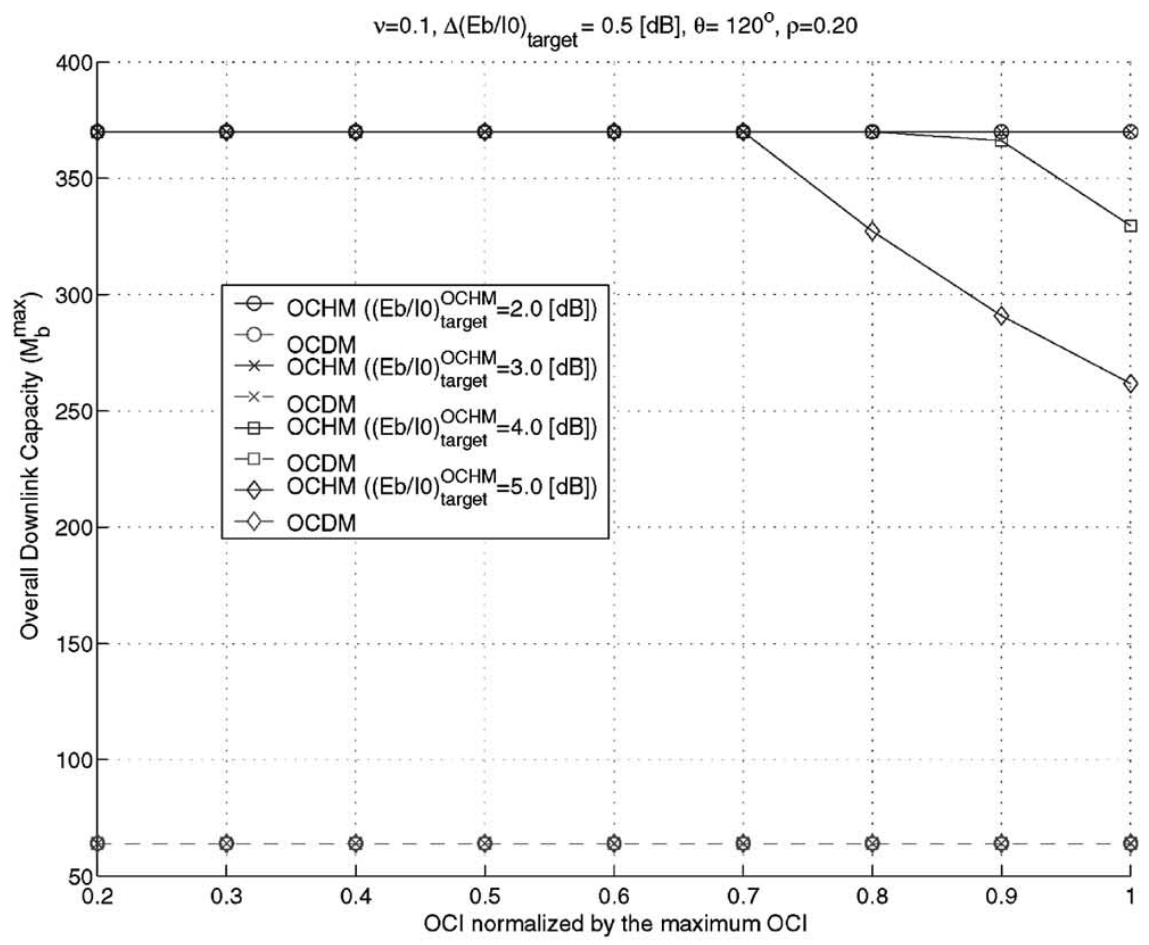

(a)

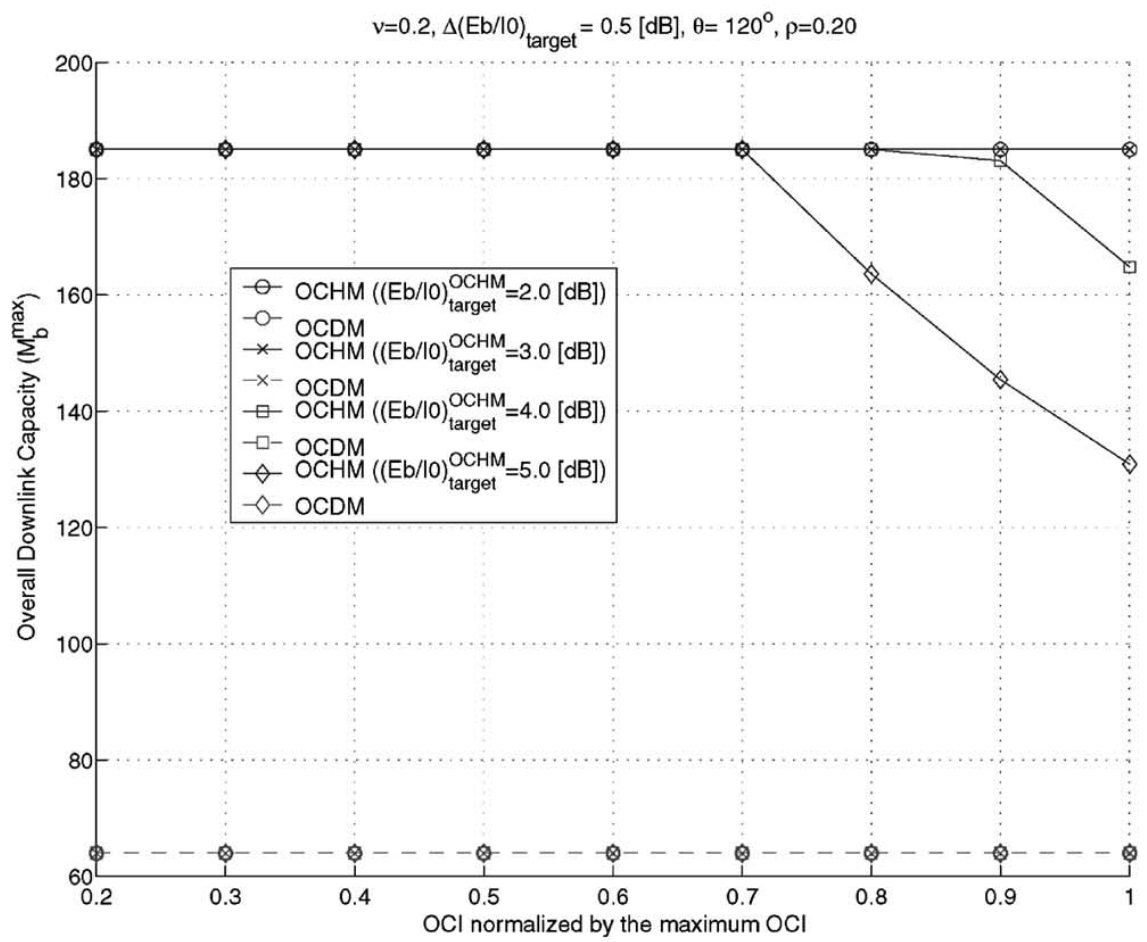

(b)

Fig. 11. Overall system capacity of OCDM and OCHM in a three-sectorized cell environment. (a) $\bar{\nu}=0.1$. (b) $\bar{\nu}=0.2$.

OCHM increases the code capacity by statistical multiplexing. The overall system capacity in OCHM is greatly improved for low-mean channel-activity values, small required $\left(E_{\mathrm{b}} / I_{0}\right)_{\text {target }}$ values, and small values of OCI. These conditions are well matched with expected future wireless communication systems since packet-based services exhibit lower mean channel activities than voice services, and enhanced modem technologies and strong channel coding schemes reduce the required $E_{\mathrm{b}} / I_{0}$ values, and advanced antenna technologies, such as the switched-beam array antenna (SBAA) system and the adaptive array antenna (AAA), reduce the amount of interference.

As further studies, we will perform feasibility studies of soft and hard handoffs in OCHM, and model the sectored antenna with real parameters. These approaches may enhance the capacity analysis of OCHM. 


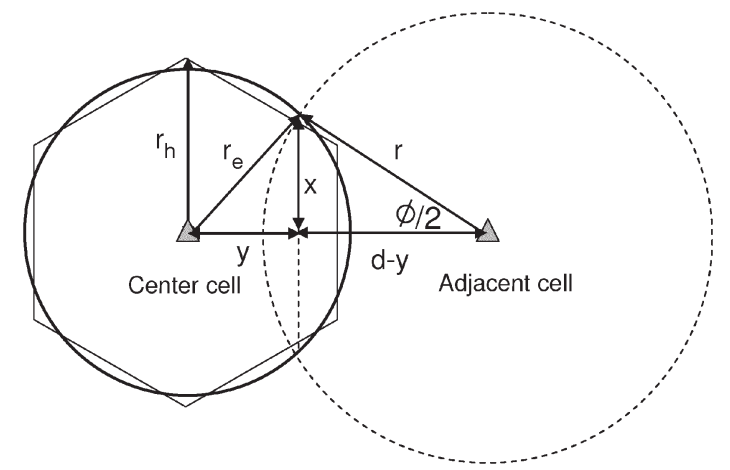

Fig. 12. Approximation of probability density function for $R_{2}$.

\section{APPENDIX}

\section{A. Probability Density Function for $R_{2}$}

We obtain the probability density function $f_{R_{2}}(r)$ of a random variable $R_{2}$ that represents the distance between a BS and an MS in a cell adjacent to the cell covered by the BS. From Fig. 12, it can be approximated as $f_{R_{2}}(r) \simeq r \phi / \pi r_{\mathrm{e}}^{2}$, where $\phi$ is the angle between two lines from an adjacent BS to two cross points of a equivalent circle of the center cell and a circle with radius $r$ from the adjacent $\mathrm{BS} . \pi r_{\mathrm{e}}^{2}$ is the area of the equivalent circle of the center cell, and $r \phi$ is the length of the arc between the two cross points.

The probability density function $f_{R_{2}}(r)$ can be approximated as

$$
f_{R_{2}}(r) \simeq \frac{r \phi}{\pi r_{\mathrm{e}}^{2}} \simeq \frac{2 x}{\pi r_{\mathrm{e}}^{2}}
$$

where $2 x$ is the distance between the two cross points.

Using a cross point, the center BS, and the adjacent BS, a triangle can be constructed, where $x$ is the length of a perpendicular line from the cross point to the base line. If we let $y$ be the distance from the center BS to a contact point between the perpendicular line and the base line, we can obtain two equations from the two triangles:

$$
\begin{aligned}
x^{2}+y^{2} & =r_{\mathrm{e}}^{2} \\
x^{2}+(d-y)^{2} & =r^{2} .
\end{aligned}
$$

From the above two equations, $x$ can be derived, $r_{\mathrm{h}} \simeq r_{\mathrm{e}}$ and $d=\sqrt{3} r_{\mathrm{h}}$, and, finally, $f_{R_{2}}(r)$ can be derived as

$$
f_{R_{2}}(r)=\frac{2}{\sqrt{3}} \cdot \frac{\sqrt{4 d^{2} r_{\mathrm{e}}^{2}-\left(d^{2}+r_{\mathrm{e}}^{2}-r^{2}\right)^{2}}}{d^{3}} .
$$

\section{B. Expectation of Total Transmission Chip Energy}

We can express the transmission chip energy from BS $b$ for MS $i, E_{\mathrm{c}, b \rightarrow(i, 0, b)}^{(\mathrm{t})}$ as a weighted sum of conditional expectation values $E\left[E_{\mathrm{c}, b \rightarrow(i, 0, b)}^{(\mathrm{t})} \mid\right.$ active $]$ and $E\left[E_{\mathrm{c}, b \rightarrow(i, 0, b)}^{(\mathrm{t})} \mid\right.$ inactive $]$.
Since $E\left[E_{\mathrm{c}, b \rightarrow(i, 0, b)}^{(\mathrm{t})} \mid\right.$ inactive $]=0$, the expectation of total transmission chip energy from a $\mathrm{BS} b$ is derived as

$$
\begin{gathered}
\sum_{(i, 0, b)} E\left[E_{\mathrm{c}, b \rightarrow(i, 0, b)}^{(\mathrm{t})}\right] \\
=\sum_{(i, 0, b)}\left(E [ E _ { \mathrm { c } , b \rightarrow ( i , 0 , b ) } ^ { ( \mathrm { t } ) } | \text { active } ] \cdot E \left[\nu_{\mathrm{c}, b \rightarrow(i, 0, b)]}\right.\right. \\
\left.+E\left[E_{\mathrm{c}, b \rightarrow(i, 0, b)}^{(\mathrm{t})} \mid \text { inactive }\right] \cdot\left(1-E\left[\nu_{\mathrm{c}, b \rightarrow(i, 0, b)}\right]\right)\right) \\
=\sum_{(i, 0, b)} E\left[E_{\mathrm{c}, b \rightarrow(i, 0, b)}^{(\mathrm{t})} \mid \text { active }\right] \cdot E\left[\nu_{\mathrm{c}, b \rightarrow(i, 0, b)}\right.
\end{gathered}
$$

where $\nu_{\mathrm{c}, b \rightarrow(i, 0, b)}$ is the channel activity of the downlink channel from BS $b$ to MS $i$.

The received transmission chip energy can be expressed with a received $\left(E_{\mathrm{c}} / I_{0}\right)_{\text {target }}$ value and interference values, and the expectation values of propagation loss and shadowing is already derived in (9) and (11). Thus, the expectation of transmission chip energy with an active condition is given by

$$
\begin{aligned}
& E\left[E_{\mathrm{c}, b \rightarrow(i, 0, b)}^{(\mathrm{t})} \mid \text { active }\right] \\
& =E\left[E_{\mathrm{c}, b \rightarrow(i, 0, b)}^{(\mathrm{r})} \mid \text { active }\right] \cdot E\left[r_{b \rightarrow(i, 0, b)}^{\gamma}\right] \cdot E\left[10^{\frac{\zeta_{b \rightarrow(i, 0, b)}}{10}}\right] \\
& =\left(\frac{E_{\mathrm{c}}}{I_{0}}\right)_{\text {target }} \cdot E\left[I_{0,(i, 0, b)}\right] \cdot E\left[r_{b \rightarrow(i, 0, b)}^{\gamma}\right] \cdot E\left[10^{\frac{\zeta_{b \rightarrow(i, 0, b)}}{10}}\right] \\
& =\left(\frac{E_{\mathrm{c}}}{I_{0}}\right)_{\text {target }} \cdot\left(E\left[I_{\mathrm{ic},(i, 0, b)}\right]+E\left[I_{\mathrm{oc},(i, 0, b)}\right]+N_{0}\right) \\
& \frac{2}{\gamma+2}\left(r_{\mathrm{e}}^{\gamma}-\varepsilon^{\gamma}\right) \cdot \exp \left\{\frac{\left(\frac{\ln 10}{10} \sigma_{\zeta}\right)^{2}}{2}\right\} \text {. }
\end{aligned}
$$

Substituting (B2), (15), and (16) in (B1), and using that the expectation values for all MS $i$ 's are identical, the expectation of total transmission chip energy is given by

$$
\begin{aligned}
\sum_{(i, 0, b)} E\left[E_{\mathrm{c}, b \rightarrow(i, 0, b)}^{(\mathrm{t})}\right] & \\
=M_{b}^{\mathrm{p}} \cdot \bar{\nu} \cdot\left(\frac{E_{\mathrm{c}}}{I_{0}}\right)_{\text {target }} & {\left[\left(\rho E_{\mathrm{c}, \max }^{(\mathrm{t})}+\sum_{\{(x, 0, b) \mid x \neq i\}} \bar{\nu} \cdot E\left[E_{\mathrm{c}, b \rightarrow(x, 0, b)}^{\mathrm{t})} \mid \text { active }\right]\right)\right.} \\
\cdot & \frac{4}{\gamma^{2}-4} \cdot \frac{\left(r_{\mathrm{e}}^{\gamma}-\varepsilon^{\gamma}\right)^{2}}{r_{\mathrm{e}}^{\gamma} \varepsilon^{\gamma}} \cdot \exp \left\{\left(\frac{\ln 10}{10} \sigma_{\zeta}\right)^{2}\right\} \cdot(1-\bar{\alpha}) \\
+ & \left\{\sum_{z \neq b}\left(\rho E_{\mathrm{c}, \max }^{(\mathrm{t})}+\sum_{(x, 0, z)} \bar{\nu} \cdot E\left[E_{\mathrm{c}, z \rightarrow(x, 0, z)}^{(\mathrm{t})} \mid \text { active }\right]\right)\right. \\
& \cdot E\left[r_{z \rightarrow(i, 0, b)}^{-\gamma}+N_{0}\right\} \\
& \left.\frac{2}{\gamma+2}\left(r_{\mathrm{e}}^{\gamma}-\varepsilon^{\gamma}\right) \cdot \exp \left\{\frac{\left(\frac{\ln 10}{10} \sigma_{\zeta}\right)^{2}}{2}\right\}\right] .
\end{aligned}
$$




\section{REFERENCES}

[1] S. Park and D. K. Sung, "Orthogonal code hopping multiplexing," IEEE Commun. Lett., vol. 6, no. 12, pp. 529-531, Dec. 2002.

[2] 3GPP, 3rd Generation Partnership Project: Technical Specification Group Radio Access Network, ser. 3GPP TS 25, Jun. 2001.

[3] TIA/EIA, Physical Layer Standard for cdma2000 Spread Spectrum Systems, 3GPP2 C.S0002, Version 3.0, Jun. 15, 2001

[4] K. Sipila, Z. Honkasalo, J. Laiho-Steffens, and A. Wacker, "Estimation of capacity and required transmission power of WCDMA downlink based on a downlink pole equation," in Proc. Vehicular Technology Conf. (VTC)-Spring, Tokyo, Japan, May 15-18, 2000, vol. 2, pp. 1002-1005.

[5] S. Burley, "Downlink capacity estimation in a WCDMA cellular network," in Proc. Personal Indoor and Mobile Radio Communications (PIMRC), San Diego, CA, Sep. 30-Oct. 3, 2001, vol. 1, pp. 26-30.

[6] W. Choi and J. Y. Kim, "Forward-link capacity of a DS/CDMA system with mixed multirate sources," IEEE Trans. Veh. Technol., vol. 50, no. 3, pp. 737-749, May 2001.

[7] S. Park and D. K. Sung, "Orthogonal code hopping multiplexing for downlink in spread spectrum communications," in Proc. MultiDimensional Mobile Communication (MDMC), Pori, Finland, Jun. 2001, pp. 365-372.

[8] S. Park, S. H. Moon, J. K. Kwon, and D. K. Sung, "Orthogonal code hopping multiplexing for downlink statistical multiplexing," in Proc. Int. Symp. Spread Spectrum Techniques and Applications (ISSSTA), Prague, Czech Republic, Sep. 2002, pp. 588-592.

[9] J. K. Kwon, S. Park, D. K. Sung, and H. Lee, "Adaptive code rate for Orthogonal Code Hopping Multiplexing (OCHM) in synchronous downlink," in Proc. Wireless Communications and Networking Conf. (WCNC), New Orleans, LA, Mar. 2003, pp. 855-859.

[10] J. K. Kwon, S. Park, and D. K. Sung, "Log-likelihood ratio (LLR) conversion schemes in orthogonal code hopping multiplexing," IEEE Commun. Lett., vol. 7, no. 3, pp. 104-106, Mar. 2003.

[11] — "Collision mitigation by Log-likelihood ratio (LLR) conversion in orthogonal code hopping multiplexing," IEEE Trans. Veh. Technol., vol. 55, no. 2, Mar. 2006.

[12] T. Onizawa and T. Hasegawa, "The spread spectrum code hopping system," in Proc. Int. Symp. Spread Spectrum Techniques and Applications (ISSSTA), Oulu, Finland, Jul. 1994, vol. 1, pp. 287-291.

[13] B. Unal and Y. Tanik, "Capacity improvement by code-hopping in S-CDMA systems," in Proc. Int. Conf. Communications (ICC), Atlanta, GA, Jun. 1998, vol. 2, pp. 989-993.

[14] $~$ "Code-hopping as a new strategy to improve performance of S-CDMA cellular systems," in Proc. Global Telecommunications (GLOBECOM), London, U.K., Nov. 18-22, 1996, vol. 2, pp. 1316-1319.

[15] J. Zander and S. Kim, Radio Resource Management for Wireless Networks. Norwood, MA: Artech House, 2001.

[16] H. Holma and A. Toskala, WCDMA for UMTS: Radio Access for Third Generation Mobile Communications. New York: Wiley, 2000.

Sung Ho Moon (S'01) received the B.S. and M.S. degrees in electrical engineering from the Korea Advanced Institute of Science and Technology (KAIST), Daejeon, Korea, in 1999 and 2001, respectively, where he is currently working toward the Ph.D. degree.

Since March 1999, he has been a Teaching and a Research Assistant in the Department of Electrical Engineering and Computer Science (EECS), KAIST. His research interests include frequency and code-hopping systems for packet data transmission, wireless scheduling algorithms, link and systemlevel simulations for $3 \mathrm{G}$ and $4 \mathrm{G}$ wireless communication systems, adaptive modulation and coding, and multiple-input multiple-output (MIMO) systems.
Suwon Park (S'92-A'03-M'04) received the B.S. degree in electrical engineering and mathematics (double major) in 1994, the M.S. degree in electrical engineering in 1996, and the Ph.D. degree in the Department of Electrical Engineering and Computer Science (EECS), all from the Korea Advanced Institute of Science and Technology (KAIST), Daejeon, Korea, in 2003.

From 1994 to 1998, he was a Research Engineer in Telecommunication R\&D Center of Samsung Electronics, where he worked for the standardization of International Mobile Telecommunications (IMT)-2000. From 1999 to 2002, he was a Research Assistant in the Department of EECS, KAIST, and a Part-Time Researcher with the Electronics and Telecommunications Research Institute (ETRI), Daejeon. In 2002, he rejoined the Telecommunication R\&D Center of Samsung Electronics as a Senior Research Engineer, where he worked for the development of terminal modems based on software-defined radio (SDR). In 2006, he joined the faculty of the Kwangwoon University, Seoul, Korea, where he is currently an Assistant Professor with the Department of Electronics and Communications Engineering. His research interests include mobile communication systems, statistical multiplexing, radio resource management, communication theory, and SDR.

Dr. Park is a member of the Institute of Electronics Engineers of Korea (IEEK) and the Korean Institute of Communication Sciences (KICS).

Jae Kyun Kwon (S'96-A'03-M'03) received the B.S., M.S., and Ph.D. degrees in electrical engineering from Korea Advanced Institute of Science and Technology (KAIST), Daejeon, Korea, in 1996, 1998, and 2003, respectively.

He is currently working for Electronics and Telecommunications Research Institute (ETRI), Daejeon. His research interests include intercell interference management, air interface for 3G evolution systems, code-hopping systems for packet data transmission, code division multiple access (CDMA) soft handoff, and indoor and urban area mobility modeling.

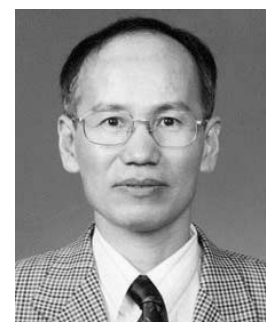

Dan Keun Sung (S'80-M'82-SM'00) received the B.S. degree in electronics engineering from Seoul National University, Seoul, Korea, in 1975 and the M.S. and Ph.D. degrees in electrical and computer engineering from the University of Texas at Austin in 1982 and 1986, respectively.

His research interests include mobile communication systems and networks, high-speed networks, next generation IP-based networks, traffic control in wireless and wireline networks, signaling networks, intelligent networks, performance and reliability of communication systems, and microsatellites. From May 1977 to July 1980, he was a Research Engineer at the Electronics ad Telecommunications Research Institute (ETRI), where he had been engaged in various projects including the development of an electronic switching system. In 1986, he joined the faculty of the Korea Advanced Institute of Science and Technology (KAIST), Daejeon, Korea, where he is currently Professor with the Department of Electrical Engineering and Computer Science (EECS). He was Director of the Satellite Technology Research Center (SaTReC) of KAIST from 1996 to 1999.

Dr. Sung is Editor of IEEE Communication Magazine. He is also Division Editor of the Journal of Communications and Networks. He was Vice-Chair of International Conference on Communications (ICC) 2002 Symposium on Global Service Portability and Infrastructure for Next Generation Virtual Home and Office Environments and Program Cochair of the Globecom 2002 Symposium on Service Infrastructure for Virtual Enterprise Environments. He was also General Conference Chair of ICC 2005 and Technical Program Program Committee Vice-Chair of ICC 2005. He received the National Order of Merits, the Dongbaek Medal in 1992, the Research Achievement Award in 1997, the MoMuc Paper Award in 1997, the Academic Excellent Award in 2000, the Best Paper Award of the Asia-Pacific Conference on Communications (APCC)2000, and This Month's Scientist Award from the Ministry of Science and Technology (MOST) and the Korea Science and Engineering Foundation (KOSEF) in 2004. He is a member of Institute of Electronics, Information, and Communications Engineers (IEICE) and the Korean Institute of Communication Sciences (KICS). He is also a member of Phi Kappa Phi and Tau Beta Pi. 